\title{
A New $k$-Distribution Scheme for Clear-Sky Radiative Transfer Calculations in Earth's Atmosphere. Part I: Thermal Infrared (Longwave) Radiation
}

\author{
MING-DAH CHOU \\ Department of Atmospheric Sciences, National Central University, Taoyuan, Taiwan \\ Jack Chung-Chieh Yu, Wei-Liang Lee, And Chein-Jung Shiu \\ Research Center for Environmental Changes, Academia Sinica, Taipei, Taiwan \\ KYU-TAE LEE AND IL-SUNG ZO \\ Department of Atmospheric and Environmental Sciences, Gangneung-Wonju National University, \\ Gangneung, South Korea \\ JOON-BUM JEE \\ Research Center for Atmospheric Environment, Hankuk University of Foreign Studies, \\ Yongin, South Korea \\ BU-YO KIM \\ Department of Atmospheric and Environmental Sciences, Gangneung-Wonju National University, \\ Gangneung, South Korea
}

(Manuscript received 10 April 2019, in final form 6 April 2020)

\begin{abstract}
A new $k$-distribution scheme of longwave radiation without the correlated-k-distribution assumption is developed. Grouping of spectral points is based on the line-by-line (LBL)-calculated absorption coefficient $k$ at a few sets of reference pressure $p_{r}$ and temperature $\theta_{r}$, where the cooling rate is substantial in a spectral band. In this new scheme, the range of $k\left(p_{r}, \theta_{r}\right)$ of a band is divided into a number of equal intervals, or $g$ groups, in $\log _{10}\left(k_{r}\right)$. A spectral point at the wavenumber $\nu$ is identified with one of the $g$ groups according to its $k_{v}\left(p_{r}, \theta_{r}\right)$. For each $g$ group, a Planck-weighted $k$-distribution function $H_{g}$ and a nonlinearly averaged absorption coefficient $\bar{k}_{g}(p, \theta)$ are derived. The function $H_{g}$ and the absorption coefficient $\bar{k}_{g}(p, \theta)$ constitute the new $k$-distribution scheme. In this $k$-distribution scheme, a spectral point can only be identified with a $g$ group regardless of pressure and temperature, which is different from the correlated- $k$ distribution scheme. The $k$-distribution scheme is applied to the $\mathrm{H}_{2} \mathrm{O}, \mathrm{CO}_{2}, \mathrm{O}_{3}, \mathrm{~N}_{2} \mathrm{O}$, and $\mathrm{CH}_{4}$ absorption bands, and results are compared with LBL calculations. To balance between the accuracy and the computational economy, the number of $g$ groups in a band of a given gas is chosen such that 1 ) the difference in cooling rate is $<0.1 \mathrm{~K} \mathrm{day}^{-1}$ in the troposphere and $<1.0 \mathrm{~K} \mathrm{day}^{-1}$ in the stratosphere and 2) the difference in fluxes is $<0.5 \mathrm{~W} \mathrm{~m}^{-2}$ at both the top of the atmosphere and the surface. These differences are attained with $130 \mathrm{~g}$ groups, which is the sum of the $g$ groups of all five gases.
\end{abstract}

\section{Introduction}

In the thermal infrared [longwave (LW)], the absorption coefficient $k$ varies rapidly with wavenumber $\nu$. Within a spectral band with a width of, say, $100 \mathrm{~cm}^{-1}$,

Corresponding author: Wei-Liang Lee, leelupin@gate.sinica. edu.tw the absorption coefficient might vary by many orders of magnitude. Accurate calculations of radiative transfer would require division of the spectral band into tens of thousands of intervals and treatment of each interval as monochromatic. This would lead to formidable computational burden if repeated calculations of atmospheric fluxes and cooling rate are required. One of the approaches to resolve this problem is the use of a $k$-distribution method 
that divides the spectral band into a small number of groups and split each of the tens of thousands of spectral intervals, that is, spectral points, into the groups. Within each group, all spectral points have a similar $k$ and can be treated as one identity in flux and cooling-rate calculations. Thus, computational burden is much reduced. Early studies using the $k$-distribution method for radiative transfer calculations include Ambartzumian (1936) and Arking and Grossman (1972).

The absorption coefficient varies with pressure and temperature, leading to variation of the $k$-distribution function with height in inhomogeneous atmospheres. This makes applications of the $k$-distribution method to radiative transfer calculations in Earth's atmosphere difficult without making simplifications or assumptions on the $k$ distribution. Chou and Arking (1980) assumed a single set of the $k$-distribution function throughout an inhomogeneous atmosphere, which were computed at a pair of reference pressure and temperature corresponding to the height where cooling is most prominent. The absorption coefficient at other pressures and temperatures were extrapolated from the reference pressure and temperature using "wing scaling," assuming the dominance of the wings of absorption lines on fluxes and cooling rate. This wing scaling of the $k$-distribution method is computationally very fast and can calculate accurately the cooling rate in the troposphere and lower stratosphere. However, the cooling-rate error increases in the upper stratosphere. Fomin (2004) developed an innovative $k$-distribution scheme for defining the spectral groups and the equivalent absorption coefficient for each group, hereafter referred to as $k_{g}$. The vertical profile of $k_{g}$ was derived by requiring that the downward flux in a spectral group is identical with the line-by-line (LBL) calculations at all atmospheric levels. This scheme is very fast with reasonably high accuracy in flux and cooling-rate calculations.

The correlated- $k$-distribution method is another approach to radiative transfer calculations in inhomogeneous atmospheres (e.g., Goody et al. 1989; Lacis and Oinas 1991; Fu and Liou 1992; Mlawer et al. 1997; Nakajima et al. 2000). This approximation assumes that spectral points of a given spectral group sorted by the magnitude of $k$ would remain in the same group regardless of pressure and temperature. While this is not strictly true, comparisons with LBL calculations have demonstrated that it can yield high accuracy in flux and cooling-rate calculations.

Besides the concern that the absorption coefficient varies with pressure and temperature when applying the $k$-distribution method to inhomogeneous atmospheres, derivation of $k_{g}$ representative of a group of spectral points is another major concern in the $k$-distribution method. Unless the number of spectral groups is large such that the range of $k_{\nu}$ in a group is small and a linearly averaged mean value can be taken as representative of the group, derivation of $k_{g}$ is not trivial. It depends not only on $k_{\nu}$ in the group but also on the absorber amount (Cusack et al. 1999). Further, the use of a single $k_{g}$ to represent a large range of $k_{\nu}$ would tend to enhance absorption when atmospheric layers are combined in flux calculations (to be elaborated in section 3). It implies that even if the transmittances are computed accurately for individual atmospheric layers, the transmittance of a path comprising multiple layers would be underestimated. Empirical adjustment for $k_{g}$ is necessary if the number of spectral groups is to be kept small.

In this study, we present an alternative approach to the $k$-distribution method applicable to inhomogeneous atmospheres, which uses neither the pressure scaling for $k_{g}$ nor the assumption of the correlation of the $k$ distributions among atmospheric layers. Section 2 provides information on the LBL calculation for constructing the $k$-distribution function, the division of the LW spectrum, and the sample atmospheres for flux and cooling-rate calculations. Section 3 describes the basic equations of the $k$-distribution scheme. Section 4 details the method of constructing the $k$-distribution function. Section 5 describes derivations of $k_{g}$ and the construction of $k_{g}$ tables. Section 6 provides results of some sample flux and cooling-rate calculations with validations against LBL calculations. Concluding remarks are given in section 7 .

\section{Line-by-line calculations, spectral bands, and sample atmospheres}

The new $k$-distribution parameterization is based on detailed LBL calculations of fluxes and cooling rate. Our LBL calculations of the absorption coefficient (Chou and Kouvaris 1986) use the Air Force Geophysical Laboratory 2012 edition of the molecular absorption parameters (Rothman et al. 2013). The molecular line shape is assumed to follow the Voigt function. Following Clough et al. (1992) and Mlawer et al. (1997), the absorption coefficient at wavenumbers $>25 \mathrm{~cm}^{-1}$ from the line center is set to zero, which is equivalent to a line cutoff of $25 \mathrm{~cm}^{-1}$. Beyond the cutoff, the absorption is treated as continuum absorption (e.g., Clough et al. 1989, 1992; Mlawer et al. 1997), which is not part of the $k$-distribution parameterization and is beyond the scope of this study. The effect of line mixing on the line shape is not included in this study. The absorption coefficient is computed at spectral intervals of $0.001 \mathrm{~cm}^{-1}$ for all gases throughout the atmosphere.

There are a number of issues concerning LBL flux and cooling-rate calculations. The spectral resolution $d v$ of 
$0.001 \mathrm{~cm}^{-1}$ may not be adequate in the middle and upper stratosphere where the line width is dominated by the Doppler broadening, which decreases linearly with decreasing wavenumber. Thus, the impact of $d v$ on flux calculations is the largest near the centers of the $25 \mu \mathrm{m}$ water vapor band $\left(20-540 \mathrm{~cm}^{-1}\right)$ and the $15 \mu \mathrm{m} \mathrm{CO}$ band $\left(540-620 \mathrm{~cm}^{-1}\right)$, where the Doppler line width is the smallest and the cooling rate in the stratosphere is large. To test the impact of $d v$ on LBL calculations, we compared the fluxes and cooling rate for these two spectral regions using $d v=0.001$ and $0.0001 \mathrm{~cm}^{-1}$ and found that the difference in the upward flux at the top of the atmosphere and the downward flux at the surface is very small with a magnitude $<0.01 \mathrm{Wm}^{-2}$. The impact on the cooling rate is also very small, $<0.02 \mathrm{~K} \mathrm{day}^{-1}$. These results demonstrate that the use of $d v=0.001 \mathrm{~cm}^{-1}$ is appropriate for flux and cooling-rate calculations.

Other issues concerning the LBL calculations include angular integrations for fluxes and the effective emitting temperature of a layer. These are issues not only in the LBL flux calculations but also in the $k$-distribution flux calculations. For the former issue, the flux transmittance is derived using the function of the exponential integral of third order, Eq. (3.15). For the latter issue, the effective emitting temperature of an atmospheric layer should vary with the optical thickness especially if the layer is thick (e.g., Clough et al. 1992; Mlawer et al. 1997; Chou and Lee 2005). Because this issue is not part of the $k$-distribution parameterization, the effective emitting temperature is set to the mean temperature of a layer for simplicity.

The thermal infrared is divided into 11 bands. Table 1 shows the spectral ranges of these bands. These bands are identical with that of Chou et al. (2003), except the 3 subbands in band 3 ( $15 \mu \mathrm{m} \mathrm{CO}_{2}$ band) is explicitly divided into 3 bands. Thus, the 9 bands in Chou et al. (2003) become 11 bands in this study. Also shown in Table 1 are the bands where this $k$-distribution scheme is applied for the five gases of $\mathrm{H}_{2} \mathrm{O}, \mathrm{CO}_{2}, \mathrm{O}_{3}, \mathrm{~N}_{2} \mathrm{O}$, and $\mathrm{CH}_{4}$. The $\mathrm{CO}$ absorption band centered at $2143 \mathrm{~cm}^{-1}$ is not included in this study. The background $\mathrm{CO}$ concentration is very small ( $\sim 100 \mathrm{ppbv})$ but might be large in urban and industrial centers with high air pollution, where the cooling due to this band may be significant.

Fluxes and cooling rate computed using the $k$-distribution parameterization for three atmospheres typical of tropical, midlatitude summer, and subarctic winter conditions, as well as two extreme, artificial atmospheres are compared with LBL calculations. Each atmosphere is divided into 75 layers. The thickness of a layer is constant in pressure, $\Delta p=24 \mathrm{hPa}$, in the troposphere, and constant in $\Delta \log _{10} p=0.15$ above the tropopause. The temperature, humidity, and ozone profiles of the
TABLE 1. Spectral bands and the number of $g$ groups $M$. The number $M$ is the result of best fits that meets the imposed accuracy requirements in flux and cooling-rate parameterizations.

\begin{tabular}{ccrccccr}
\hline \hline \multirow{2}{*}{$\begin{array}{c}\text { Spectral } \\
\text { band }\end{array}$} & $\begin{array}{c}\text { Spectral } \\
\text { region }\left(\mathrm{cm}^{-1}\right)\end{array}$ & \multicolumn{5}{c}{ Number of g groups $M$} \\
\cline { 3 - 7 } & $\mathrm{H}_{2} \mathrm{O}$ & $\mathrm{CO}_{2}$ & $\mathrm{O}_{3}$ & $\mathrm{~N}_{2} \mathrm{O}$ & $\mathrm{CH}_{4}$ & All \\
\hline 1 & $20-340$ & 12 & & & & & 12 \\
2 & $340-540$ & 11 & & & & & 11 \\
3 & $540-620$ & 8 & 9 & & 3 & & 20 \\
4 & $620-720$ & 6 & 9 & & & & 15 \\
5 & $720-800$ & 5 & 9 & & & & 14 \\
6 & $800-980$ & 3 & 3 & & & & 6 \\
7 & $980-1100$ & 3 & 3 & 7 & & & 13 \\
8 & $1100-1215$ & 4 & & & & & 4 \\
9 & $1215-1380$ & 7 & & & 4 & 5 & 16 \\
10 & $1380-1900$ & 8 & & & & & 8 \\
11 & $1900-3000$ & 5 & 6 & & & & 11 \\
All & $20-3000$ & 72 & 39 & 7 & 7 & 5 & 130 \\
\hline
\end{tabular}

three typical atmospheres are taken from McClatchey et al. (1972), except the specific humidity in the stratosphere is set to $3.25 \times 10^{-6}$ for the tropical atmosphere, and $4 \times 10^{-6}$ for the midlatitude summer and subarctic winter atmospheres. The concentrations of $\mathrm{CO}_{2}, \mathrm{~N}_{2} \mathrm{O}$ and $\mathrm{CH}_{4}$ based on the observations in 2014 at Mauna Loa Observatory, Hawaii, are, respectively, set to 400, 0.32 , and $1.80 \mathrm{ppmv}$, independent of height. For independent validation of the $k$-distribution approximation scheme, eight atmospheres in four diversified climatic regimes and two seasons were randomly sampled from ERA5 (Copernicus Climate Change Service 2017).

\section{The Planck-weighted $k$ distribution}

One of the critical terms in the LW radiative transfer calculations is the radiance emission of an atmospheric layer in the vertical $I$, which can be expressed as

$$
I_{\Delta \nu}=\int_{\Delta \nu} B_{\nu}(\theta)\left[1-e^{-k_{\nu}(p, \theta) \Delta u}\right] d \nu
$$

where $B$ is the Planck (blackbody) radiance, $k$ is the absorption coefficient, $\nu$ is the wavenumber, $\Delta \nu$ is the width of a spectral band, and $p, \theta$, and $\Delta u$ are, respectively, the pressure, temperature, and absorber amount of the layer.

The most detailed calculations of absorption/emission are the LBL calculation, which divides the spectrum into a large number of small intervals $N$ with a width $\delta \nu$ so that the absorption can be considered constant within $\delta \nu$. Equation (3.1) then becomes

$$
I_{\Delta \nu}=\sum_{i}^{N} I_{i}=\sum_{i}^{N} B_{i}(\theta)\left[1-e^{-k_{i}(p, \theta) \Delta u}\right] \delta \nu,
$$

where the subscript $i$ denotes the spectral point. 
The $k$-distribution approximation is to split all the spectral points into a number of groups, say $M$, such that the magnitude of $k$ is similar among the points in each of the $M$ groups. Points in the same group are treated as one identity in radiation calculations. Using this approximation, the total radiance of the gth group $I_{g}$ is given by

$$
\begin{aligned}
I_{g} & =\sum_{i \in R_{g}} B_{i}(\theta)\left[1-e^{-k_{i}(p, \theta) \Delta u}\right] \delta v \\
& \approx B_{g}(\theta)\left[1-e^{-k_{g}(p, \theta) \Delta u}\right], \quad g=1,2, \ldots, M,
\end{aligned}
$$

where $R_{g}$ is the set of $n_{g}$ spectral points of the gth group. Each spectral point can only belong to a single group:

$$
N=\sum_{g=1}^{M} n_{g}
$$

$B_{g}(\theta)$ is the total of the blackbody radiance emission at all spectral points in the gth group,

$$
B_{g}(\theta)=\sum_{i \in R_{g}} B_{i}(\theta) \delta \nu, \quad g=1,2, \ldots, M ;
$$

and $k_{g}$ is the equivalent absorption coefficient representing the effective absorption strength of all spectral points in the gth group. Finally, the radiance in the spectral band emitted by the atmospheric layer is

$$
I_{\Delta \nu}=\sum_{g=1}^{M} I_{g}=\sum_{g=1}^{M} B_{g}(\theta)\left[1-e^{-k_{g}(p, \theta) \Delta u}\right] .
$$

If the ratio of $B_{g}(\theta)$ to the total radiance of the spectral band $B_{\Delta \nu}(\theta)$ does not substantially change with temperature, Eq. (3.6) can be written as

$$
\begin{aligned}
I_{\Delta \nu} & =B_{\Delta \nu}(\theta) \sum_{g=1}^{M}\left[\frac{B_{g}(\theta)}{B_{\Delta \nu}(\theta)}\right]\left[1-e^{-k_{g}(p, \theta) \Delta u}\right] \\
& \approx B_{\Delta \nu}(\theta) \sum_{g=1}^{M}\left[\frac{B_{g}\left(\theta_{r}\right)}{B_{\Delta \nu}\left(\theta_{r}\right)}\right]\left[1-e^{-k_{g}(p, \theta) \Delta u}\right] \\
& =B_{\Delta \nu}(\theta) \sum_{g=1}^{M} H_{g}\left[1-e^{-k_{g}(p, \theta) \Delta u}\right],
\end{aligned}
$$

where $\theta_{r}$ is the reference temperature chosen to be $250 \mathrm{~K}$, and

$$
\begin{aligned}
B_{\Delta \nu}(\theta) & =\sum_{i=1}^{N} B_{i}(\theta) \delta \nu=\sum_{g=1}^{M} B_{g}(\theta), \\
H_{g} & =\frac{B_{g}\left(\theta_{r}\right)}{B_{\Delta \nu}\left(\theta_{r}\right)}=\frac{n_{g} \overline{B_{g}\left(\theta_{r}\right)}}{N \overline{B_{\Delta \nu}\left(\theta_{r}\right)}}, \quad g=1,2, \ldots, M,
\end{aligned}
$$

where the overbar denotes the mean over spectral points.

If the spectral variation in the Planck function is small within $\Delta \nu$, we have $\overline{B_{g}\left(\theta_{r}\right)} \approx \overline{B_{\Delta \nu}\left(\theta_{r}\right)}$, and Eq. (3.7) reduces to

$$
I_{\Delta \nu}=B_{\Delta \nu}(\theta) \sum_{g=1}^{M} G_{g}\left[1-e^{-k_{g}(p, \theta) \Delta u}\right]
$$

where

$$
G_{g}=\frac{n_{g}}{N}, \quad g=1,2, \ldots, M .
$$

The function $G$ is commonly defined as the $k$-distribution function. The function $H$ is then defined as the Planck-weighted $k$-distribution function (e.g., Chou and Lee 2005). The advantage of using Eq. (3.7) over Eq. (3.6) is that we need to evaluate only once for $B_{\Delta \nu}(\theta)$ instead of $M$ times for $B_{g}(\theta)$. It is noticed that values of $H$ are precomputed and fixed.

The above analyses and the definitions of $k_{g}$ and $H_{g}$ are used to derive the formulas for flux calculations using the Planck-weighted $k$-distribution approximation. For a black surface, the upward radiance at the pressure level $p_{l}$, wavenumber $\nu$, and in the direction $\mu$ is given by (Liou 2002)

$I_{\nu}^{\uparrow}\left(\mu, p_{l}\right)=B_{\nu}\left(p_{s}\right) e^{-\tau_{\nu}\left(p_{s}, p_{l}\right) / \mu}+\int_{p_{s}}^{p_{l}} B_{\nu}(p)\left[\frac{d e^{-\tau_{v}\left(p, p_{l}\right) / \mu}}{d p}\right] d p$,

where $\mu$ is cosine of the zenith angle, $p_{s}$ is the surface pressure, and $\tau$ is the optical thickness,

$$
\tau_{\nu}\left(p, p_{l}\right)=\int_{p}^{p_{l}} k_{\nu}\left(p^{\prime}, \theta^{\prime}\right)\left(\frac{d u}{d p^{\prime}}\right) d p^{\prime}
$$

Integrating over the spectral band and angles, the upward flux using the LBL method becomes

$$
\begin{aligned}
F_{\Delta \nu}^{\uparrow}\left(p_{l}\right)= & \int_{\Delta \nu}\left\{\pi B_{\nu}\left(p_{s}\right) T_{\nu}\left(p_{s}, p_{l}\right)\right. \\
& \left.+\int_{p_{s}}^{p_{l}} \pi B_{\nu}(p)\left[\frac{d T_{\nu}\left(p, p_{l}\right)}{d p}\right] d p\right\} d \nu,
\end{aligned}
$$

where $T$ is the diffuse transmittance given by

$$
T_{\nu}\left(p, p_{l}\right)=2 \int_{0}^{1} e^{-\tau_{\nu}\left(p, p_{l}\right) / \mu} \mu d \mu
$$

Similarly, the downward flux using the LBL method is given by 


$$
F_{\Delta \nu}^{\downarrow}\left(p_{l}\right)=\int_{\Delta \nu}\left\{\int_{0}^{p_{l}} \pi B_{\nu}(p)\left[\frac{d T_{\nu}\left(p, p_{l}\right)}{d p}\right] d p\right\} d \nu .
$$

In the $k$-distribution approximation, the upward radiance in the gth group is derived by integrating Eq. (3.12) over all spectral points of the group,

$$
\begin{aligned}
I_{g}^{\uparrow}\left(\mu, p_{l}\right)= & \sum_{i=\in R_{g}} I_{\nu}^{\uparrow}\left(\mu, p_{l}\right) \\
= & H_{g}\left\{B_{\Delta \nu}\left(p_{s}\right) e^{-\tau_{g}\left(p_{s}, p_{l}\right) / \mu}+\int_{p_{s}}^{p_{l}} B_{\Delta \nu}(p)\right. \\
& \left.\times\left[\frac{d e^{-\tau_{g}\left(p, p_{l}\right) / \mu}}{d p}\right] d p\right\}, g=1,2, \ldots, M,
\end{aligned}
$$

where

$$
\begin{gathered}
H_{g} B_{\Delta \nu}(p)=\sum_{i \in R_{g}} B_{i}(p) \delta \nu=B_{g}(p), \\
\tau_{g}\left(p, p_{l}\right)=\int_{p}^{p_{l}} k_{g}\left(p^{\prime}, \theta^{\prime}\right)\left(\frac{d u}{d p^{\prime}}\right) d p^{\prime} .
\end{gathered}
$$

By angular integration of $I_{g}$, the upward flux is given by

$$
\begin{aligned}
F_{g}^{\uparrow}\left(p_{l}\right)= & H_{g}\left\{\pi B_{\Delta \nu}\left(p_{s}\right) T_{g}\left(p_{s}, p_{l}\right)+\int_{p_{s}}^{p_{l}} \pi B_{\Delta \nu}(p)\right. \\
& \left.\times\left[\frac{d T_{g}\left(p, p_{l}\right)}{d p}\right] d p\right\}, \quad g=1,2, \ldots, M,
\end{aligned}
$$

and the diffuse transmittance is

$$
T_{g}\left(p, p_{l}\right)=2 \int_{0}^{1} e^{-\tau_{g}\left(p, p_{l}\right) / \mu} \mu d \mu
$$

To expedite radiative transfer calculations, the angular integration for the diffuse transmittance is simplified by the use of a single angle $\phi$,

$$
T_{g}\left(p, p_{l}\right) \approx e^{-\bar{\tau}_{g}\left(p, p_{l}\right)}
$$

where

$$
\bar{\tau}_{g}\left(p, p_{l}\right)=\int_{p_{l}}^{p} \bar{k}_{g}\left(p^{\prime}, \theta^{\prime}\right)\left(\frac{d u}{d p^{\prime}}\right) d p^{\prime}
$$

and

$$
\bar{k}_{g}(p, \theta)=k_{g}(p, \theta) / \cos \left(\phi_{g}\right) .
$$

The parameter $1 / \cos \left(\phi_{g}\right)$ is commonly called the diffusivity factor (Liou 2002), and $\bar{k}_{g}(p, \theta)$ is called the diffusivity-amplified equivalent absorption coefficient in this study.

Similarly, the downward flux is given by

$$
\begin{aligned}
F_{g}^{\downarrow}\left(p_{l}\right) & =H_{g}\left\{\int_{0}^{p_{l}} \pi B_{\Delta \nu}(p)\left[\frac{d T_{g}\left(p, p_{l}\right)}{d p}\right] d p\right\}, \\
g & =1,2, \ldots, M .
\end{aligned}
$$

Integration over all $g$ groups, the upward and downward fluxes in a band $\Delta \nu$ using the $k$-distribution method are

$$
\begin{aligned}
F_{\Delta \nu}^{\uparrow}\left(p_{l}\right)= & \pi B_{\Delta \nu}\left(p_{s}\right) T_{\Delta \nu}\left(p_{s}, p_{l}\right) \\
& +\int_{p_{s}}^{p_{l}} \pi B_{\Delta \nu}(p)\left[\frac{d T_{\Delta \nu}\left(p, p_{l}\right)}{d p}\right] d p
\end{aligned}
$$

and

$$
F_{\Delta \nu}^{\downarrow}\left(p_{l}\right)=\int_{0}^{p_{l}} \pi B_{\Delta \nu}(p)\left[\frac{d T_{\Delta \nu}\left(p, p_{l}\right)}{d p}\right] d p,
$$

where $T_{\Delta \nu}$ is the Planck-weighted diffuse transmittance of a band given by

$$
T_{\Delta \nu}=\sum_{g=1}^{M} H_{g} T_{g} .
$$

Finally, for a nonblack surface, the upward flux needs to be adjusted by reducing the surface emission and adding the reflection of radiation by the surface. For the LBL calculation, Eq. (3.14) becomes

$$
\begin{aligned}
F_{\Delta \nu}^{\uparrow}\left(p_{l}\right)= & \int_{\Delta \nu} d \nu\left\{\left[\varepsilon_{\nu} \pi B_{\nu}\left(p_{s}\right)+\left(1-\varepsilon_{\nu}\right) F_{\nu}^{\downarrow}\left(p_{s}\right)\right] T_{\nu}\left(p_{s}, p_{l}\right)\right. \\
& \left.+\int_{p_{s}}^{p_{l}} \pi B_{\nu}(p)\left[\frac{d T_{\nu}\left(p, p_{l}\right)}{d p}\right] d p\right\},
\end{aligned}
$$

where $\varepsilon_{\nu}$ is the surface emissivity, $T_{\nu}\left(p_{s}, p_{l}\right)$ is given by Eq. (3.15), and the downward flux at the surface is given by

$$
F_{\nu}^{\downarrow}\left(p_{s}\right)=\int_{0}^{p_{s}} \pi B_{\nu}(p)\left[\frac{d T_{\nu}\left(p, p_{s}\right)}{d p}\right] d p .
$$

For a nonblack surface using the $k$-distribution method, Eq. (3.26) becomes

$$
\begin{aligned}
F_{\Delta \nu}^{\uparrow}\left(p_{l}\right)= & {\left[\varepsilon_{\Delta \nu} \pi B_{\Delta \nu}\left(p_{s}\right)+\left(1-\varepsilon_{\Delta \nu}\right) F_{\Delta \nu}^{\downarrow}\left(p_{s}\right)\right] T_{\Delta \nu}\left(p_{s}, p_{l}\right) } \\
& +\int_{p_{s}}^{p_{l}} \pi B_{\Delta \nu}(p)\left[\frac{d T_{\Delta \nu}\left(p, p_{l}\right)}{d p}\right] d p
\end{aligned}
$$


where $\varepsilon_{\Delta \nu}$ is the mean surface emissivity of a spectral band, $T_{\Delta \nu}\left(p_{s}, p_{l}\right)$ is given by Eqs. (3.22) and (3.28), and $F_{\Delta \nu}^{\downarrow}\left(p_{s}\right)$ is given by Eq. (3.27). In this study, the surface is assumed to be black, and the emissivity $\varepsilon$ is set to 1 .

In the $k$-distribution approximation, the transmission of a $g$ group is calculated from Eq. (3.22), where the diffusivity-amplified equivalent absorption coefficient is used to compute the optical thickness of the $g$ group. It is equivalent to the approximation of nongray radiation of a group of spectral points by gray radiation, that is, by using a single absorption coefficient. This approximation would inevitably introduce uncertainties in the transmission of a $g$ group. For the case of combining neighboring layers, the mean transmission of a band (or a $g$ group) is given by

$$
\overline{T_{12}}=\frac{1}{\Delta \nu} \int_{\Delta \nu} T_{1, \nu} T_{2, \nu} d \nu=\overline{T_{1}} \overline{T_{2}}+\frac{1}{\Delta \nu} \int_{\Delta \nu} T_{1, \nu}^{\prime} T_{1, \nu}^{\prime} d \nu,
$$

where the subscripts 1 and 2 denote two neighboring layers, the overbar denotes band mean, and the "prime" denotes the deviation from the mean,

$$
T_{\nu}=\bar{T}+T_{\nu}^{\prime} .
$$

With the transmission of the $g$ group given by Eq. (3.22) and the optical thickness given by Eq. (3.23), it effectively assumes the following multiplication rule,

$$
\overline{T_{12}}=\overline{T_{1}} \overline{T_{2}},
$$

and the last term in Eq. (3.32) is ignored. Because the spectral variations of the transmission function between neighboring layers are highly correlated, the last term in Eq. (3.32) is positive, and the transmission using Eq. (3.34) would underestimate the transmission, or overestimate the absorption, when layers are combined. It follows that empirical adjustments for the absorption coefficient are necessary for reducing the uncertainty in flux calculations.

For the case of multiple gases with overlapping absorption, absorption between the gases can be assumed uncorrelated, and the last term in Eq. (3.32) vanishes. Thus, the multiplication rule can be applied, and Eq. (3.28) becomes (cf. Chou et al. 2003)

$$
T_{\Delta \nu}=\prod_{i} T_{\Delta \nu, i}=\prod_{i}\left(\sum_{g=1}^{M_{i}} H_{g, i} T_{g, i}\right),
$$

where the subscript $i$ denotes the absorbing gas.

Because the absorption coefficient is a function of pressure and temperature, grouping of spectral points into a small number of $g$ groups is dependent upon the choice of the reference pressure and temperature, $p_{r}$ and $\theta_{r}$. For flux calculations using Eqs. (3.27) and (3.31) with the band-mean transmittance given by Eq. (3.28) or (3.35) for overlapping absorption, the major components in the construction of the Planck-weighted $k$-distribution scheme include (i) grouping of the spectral points by the selections of $p_{r}, \theta_{r}$, and $M$; (ii) derivation of $H_{g}$; and (iii) derivation of $\bar{k}_{g}(p, \theta)$. These components are discussed in the following sections.

\section{Grouping of spectral points}

\section{a. Selection of the reference pressure and temperature}

In the $k$-distribution approximation, sorting of spectral points into a small number of groups is carried out at a set of reference pressures and temperatures $\left(p_{r}, \theta_{r}\right)$. Because the absorption coefficient varies with pressure and temperature, the absorption coefficient of those spectral points in a given group will scatter and become less packed as $(p, \theta)$ departs from $\left(p_{r}, \theta_{r}\right)$. Therefore, calculations of the transmission function are more accurate at heights where $(p, \theta)$ is close to $\left(p_{r}, \theta_{r}\right)$. Away from those heights, flux and cooling-rate calculations are less accurate. To obtain a maximal accuracy, the reference $\left(p_{r}, \theta_{r}\right)$ should be chosen at heights where cooling is most prominent (Chou and Arking 1980; Fomin 2004).

For spectral bands with weak absorption, the cooling usually peaks in the troposphere, and one pair of $\left(p_{r}, \theta_{r}\right)$ that is representative of middle tropospheric conditions would be sufficient, as the pressure range in the troposphere is not overly large. For spectral bands, on the other hand, where cooling in the stratosphere is prominent, for example the $9.6 \mu \mathrm{m} \mathrm{O}_{3}$ band, it requires at least one pair of $\left(p_{r}, \theta_{r}\right)$ presenting the middlestratospheric conditions. For spectral bands with cooling peaks in both the stratosphere and troposphere, for example, the $15 \mu \mathrm{m} \mathrm{CO} \mathrm{CO}_{2}$ band and the centers of the $\mathrm{H}_{2} \mathrm{O}$ bands, at least one pair of $\left(p_{r}, \theta_{r}\right)$ should be chosen in the stratosphere and another in the troposphere. This is because pressures and, hence, the absorption coefficients in the troposphere and stratosphere differ by many orders of magnitude. Furthermore, the Doppler broadening of the molecular absorption line becomes important in layers above the $5 \mathrm{hPa}$ level. The absorption coefficients of a group of spectral points packed together at a reference pressure in the stratosphere are likely to scatter over a wider range of values in a tropospheric layer, and vice versa.

Based on the LBL-calculated cooling-rate profiles of some typical atmospheres, the levels of peak cooling are identified, and the pressures corresponding to those levels are chosen as the reference pressures. The levels 

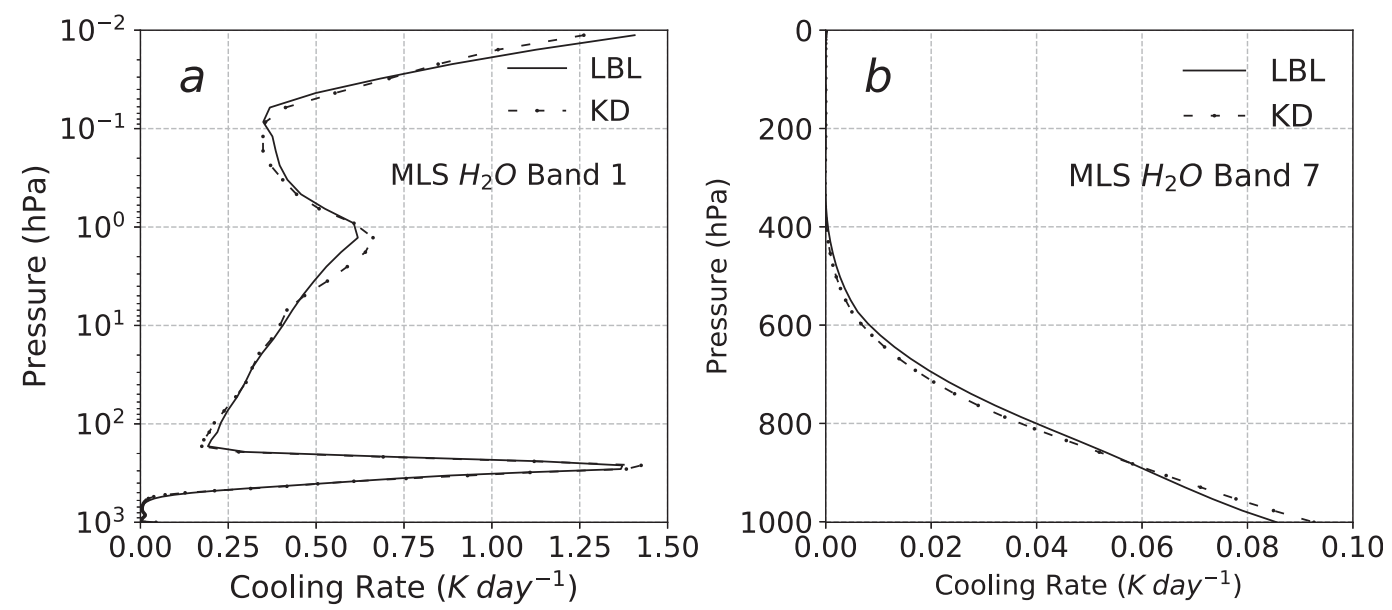

FIG. 1. Cooling-rate profiles in (left) water vapor band $1\left(20-340 \mathrm{~cm}^{-1}\right)$ and (right) water vapor band 7 (980$1100 \mathrm{~cm}^{-1}$ ) computed using the line-by-line method (LBL) and the $k$-distribution parameterization (KD) for the midlatitude summer atmosphere.

of peak cooling vary with atmospheres, but flux calculations are not sensitive to the exact value of $p_{r}$. Using two reference pressures, one in the troposphere and the other in the stratosphere, is usually adequate for grouping spectral points. It rarely requires three reference pressures. In this study, we used the cooling-rate profile of the midlatitude summer atmosphere for determining whether the reference pressure should be chosen in either the troposphere, or the stratosphere, or both. It is noticed that exact values of the reference pressure are not critical. Instead of the midlatitude summer atmosphere, other typical atmospheres can also be used. Because the absorption coefficient varies slowly within the range of the atmospheric temperature, $\theta_{r}$ is fixed at $250 \mathrm{~K}$.

Figure 1 is an example demonstrating the bases on which the reference pressures are chosen. The left panel is the cooling-rate profiles computed for the center of the water vapor absorption band, band $1\left(20-340 \mathrm{~cm}^{-1}\right)$. Two cooling-rate profiles are demonstrated in the figure; LBL calculations using Eqs. (3.14)-(3.16) and the $k$-distribution approximation (KD) using Eqs. (3.26) and (3.27). It is one of the two bands among all the bands of the five gases in this study that requires three reference pressures, chosen at $p_{r}=1,30$, and $300 \mathrm{hPa}$. It also has the largest number of $g$ groups, $M=12$, among all of the bands and gases. As opposed to the case of band 1, the right panel demonstrates the case of the weakest water vapor absorption band, band $7\left(980-1100 \mathrm{~cm}^{-1}\right)$. The cooling is weak and attains a peak at the surface. It requires only one reference pressure somewhere in the middle troposphere, $p_{r}=600 \mathrm{hPa}$, and three $g$ groups, $M=3$. Thus, the flux and cooling-rate calculations are 4 times faster than band 1 .

\section{b. Spectral groups}

The magnitude of the absorption coefficient varies by many orders within a narrow spectral band, and the absorption coefficient in a $g$ group might range by a factor of 5 or more, depending on the number of groups $M$. Based on the fact that the transmittance is a highly nonlinear function of the absorption coefficient $k_{\nu}$, we divided the range of $k_{\nu}\left(p_{r}, \theta_{r}\right)$ of a band into $M$ intervals with a constant size of $\Delta \log _{10}\left[k_{\nu}\left(p_{r}, \theta_{r}\right)\right]$. A spectral point is identified with one of the $M$ groups according to its $k_{\nu}\left(p_{r}, \theta_{r}\right)$ value. In this way, the $g$ groups have more comparable ranges of transmittance values than if a constant size of $\Delta k_{\nu}\left(p_{r}, \theta_{r}\right)$ is used.

Grouping of spectral points involves the following three parameters. These parameters are judicially determined to obtain an optimal balance between accuracy and speed in flux and cooling-rate calculations:

- The total number of $g$ groups, $M$. The smaller the $M$, the less accurate but faster the flux calculations.

- The number of $p_{r}$ for sorting spectral points, $J$. Noticed that $\theta_{r}$ is fixed at $250 \mathrm{~K}$.

- The number of $g$ groups $m_{j}$ corresponding to each $p_{r, j}$. The subscript $j$ denotes the reference pressure. It is either 1 or 2 , and rarely 3 . All $m$ add up to $M$,

$$
M=\sum_{j=1}^{J} m_{j}, \quad J=1,2, \text { or } 3 .
$$

Steps for splitting spectral points to the $M g$ groups are summarized as follows:

1) The absorption coefficient at the $J$ sets of $p_{r}$ and $\theta_{r}(=250 \mathrm{~K})$ are computed using the LBL method. 
Results are stored in $k_{\nu}\left(p_{r, j}, \theta_{r}\right)$, where $j$ ranges from 1 to 3 .

2) Initially a large number of groups, $M=20$, is chosen.

3) Starting from the smallest reference pressure $p_{r 1}$, the maximum and minimum values of $k_{\nu}\left(p_{r 1}, \theta_{r}\right)$ in the band $\Delta \nu$ are identified, and a constant size of $\Delta x\left(p_{r 1}, \theta_{r}\right)$ for the $M$ groups is computed from

$$
\Delta x_{\nu}\left(p_{r 1}, \theta_{r}\right)=\frac{x^{\max }\left(p_{r 1}, \theta_{r}\right)-x^{\min }\left(p_{r 1}, \theta_{r}\right)}{M},
$$

where $x_{\nu}\left(p_{r 1}, \theta_{r}\right)=\log _{10}\left[k_{\nu}\left(p_{r 1}, \theta_{r}\right)\right]$, and $x^{\max }$ and $x^{\mathrm{min}}$ are the maximum and minimum of $x_{\nu}$ in the band $\Delta \nu$.

4) The upper and lower bounds of each of the $M$ groups, $x^{U_{j}}\left(p_{r 1}, \theta_{r}\right)$ and $x^{L_{j}}\left(p_{r 1}, \theta_{r}\right), j=1,2, \ldots$, $M$, are derived, where $x^{L_{j}}\left(p_{r 1}, \theta_{r}\right)=x^{U_{j}}\left(p_{r 1}, \theta_{r}\right)-$ $\Delta x_{\nu}\left(p_{r 1}, \theta_{r}\right)$. It is noticed that the grouping is in descending order of the absorption coefficient, that is, $x^{U_{j}}\left(p_{r 1}, \theta_{r}\right)>x^{U_{j+1}}\left(p_{r 1}, \theta_{r}\right)$.

5) Each spectral point is assigned to one of the $M$ groups $g_{1}(\nu)$, where $g_{1}$ ranges from 1 to $M$. This completes spectral grouping corresponding to the first reference pressure $p_{r 1}$.

6) Using a typical atmosphere, such as the midlatitude summer atmosphere, cooling-rate profiles of the $M$ groups are calculated using the LBL method, and the first $m_{1}$ cooling-rate profiles that contribute most to the cooling at $p_{r 1}$ are identified.

7) For grouping the spectral points associated with the second reference pressure $p_{r 2}$, the spectral points that have already been assigned to the first $m_{1}$ groups associated with $p_{r 1}$ are deleted from $k_{\nu}\left(p_{r 2}, \theta_{r}\right)$.

8) The maximum and minimum values of $k_{\nu}\left(p_{r 2}, \theta_{r}\right)$, are identified, and a constant size of $\Delta x_{\nu}\left(p_{r 2}, \theta_{r}\right)$ for the $\left(M-m_{1}\right)$ groups is computed from

$$
\Delta x_{\nu}\left(p_{r 2}, \theta_{r}\right)=\frac{x^{\max }\left(p_{r 2}, \theta_{r}\right)-x^{\min }\left(p_{r 2}, \theta_{r}\right)}{M-m_{1}} .
$$

9) The upper and lower bounds of each of the $\left(M-m_{1}\right)$ groups, $x^{U_{j}}\left(p_{r 2}, \theta_{r}\right)$ and $x^{L_{j}}\left(p_{r 2}, \theta_{r}\right), j=1,2, \ldots$, $M-m_{1}$, are derived, where $x^{L_{j}}\left(p_{r 2}, \theta_{r}\right)=$ $x^{U_{j}}\left(p_{r 2}, \theta_{r}\right)-\Delta x_{\nu}\left(p_{r 2}, \theta_{r}\right)$.

10) Each spectral point is assigned to one of the $\left(M-m_{1}\right)$ groups $g_{2}(\nu)$, where $g_{2}$ ranges from 1 to $\left(M-m_{1}\right)$. Again, the grouping is in descending order of the absorption coefficient.

11) Cooling-rate profiles of the $\left(M-m_{1}\right)$ groups are calculated using the LBL method, and the first $m_{2}$ cooling-rate profiles that contribute most to the cooling at $p_{r 2}$ is identified.
12) Steps 7-10 are repeated and $g_{3}(\nu)$ derived if $J=3$. It is noticed that

$$
\Delta x_{\nu}\left(p_{r 3}, \theta_{r}\right)=\frac{x^{\max }\left(p_{r 3}, \theta_{r}\right)-x^{\min }\left(p_{r 3}, \theta_{r}\right)}{M-m_{1}-m_{2}},
$$

where $\left(M-m_{1}-m_{2}\right)=m_{3}$

13) Finally, each spectral point in $\Delta \nu$ is assigned to a $g$ group $g(\nu)$ by combining the first $m_{1}$ groups of $g_{1}(\nu), m_{2}$ groups of $g_{2}(\nu)$, and $m_{3}$ groups of $g_{3}(\nu)$.

The number $M=20$ is more than adequate for accurate flux and cooling-rate calculations. By reducing $M$, the above steps are repeated, and the values of $m_{j}$ are reduced accordingly. The numbers of $M$ given in Table 1 are the "best fit" that meet the imposed accuracy requirements in flux and cooling-rate calculations using the $k$-distribution scheme. Figure 2 is a flowchart summarizing the steps described above.

\section{c. The Planck-weighted $k$-distribution function}

With each $\nu$ identified with a group $g(\nu)$, the Planckweighted $k$-distribution function $H_{g}$ are computed from Eqs. (3.5), (3.8), and (3.9), where $g=1,2,3, \ldots, M$. It is noticed that the $M$ groups are defined by the upper and lower bounds of the absorption coefficient of each $g$ group as described in steps 4 and 9 of section $4 \mathrm{~b}$. The Planck-weighted $k$-distribution function is then derived from Eq. (3.9). It is different from the correlated- $k$ distribution scheme (e.g., Fu and Liou 1992; Mlawer et al. 1997) that the upper and lower bounds defining a $g$ group are empirically specified.

\section{Equivalent absorption coefficient and precomputed $\boldsymbol{k}$ tables}

Accuracy of the $k$-distribution scheme depends strongly on how well the equivalent absorption coefficient of a $g$ group is derived, which is sensitive to the number of $g$ groups $M$ and the reference pressure $p_{r}$ on which the spectral points are grouped. Except for weak absorption, the transmission function is highly nonlinear with respect to the absorption coefficient $k_{\nu}$, and an equivalent absorption coefficient $k_{g}$ representing a group of widely scattered absorption coefficients can only be derived empirically.

For cases of weak absorption, the transmission function in Eq. (3.2) can be approximated by $\left[1-k_{i}(p, \theta) \Delta u\right]$, and from Eq. (3.3) we have

$$
k_{g, \text { lin }}(p, \theta)=\frac{1}{B_{g}(\theta)} \sum_{i \in S_{g}} B_{i}(\theta) k_{i}(p, \theta) \delta \nu,
$$

where the subscript lin denotes the linear approximation for the transmission function. 


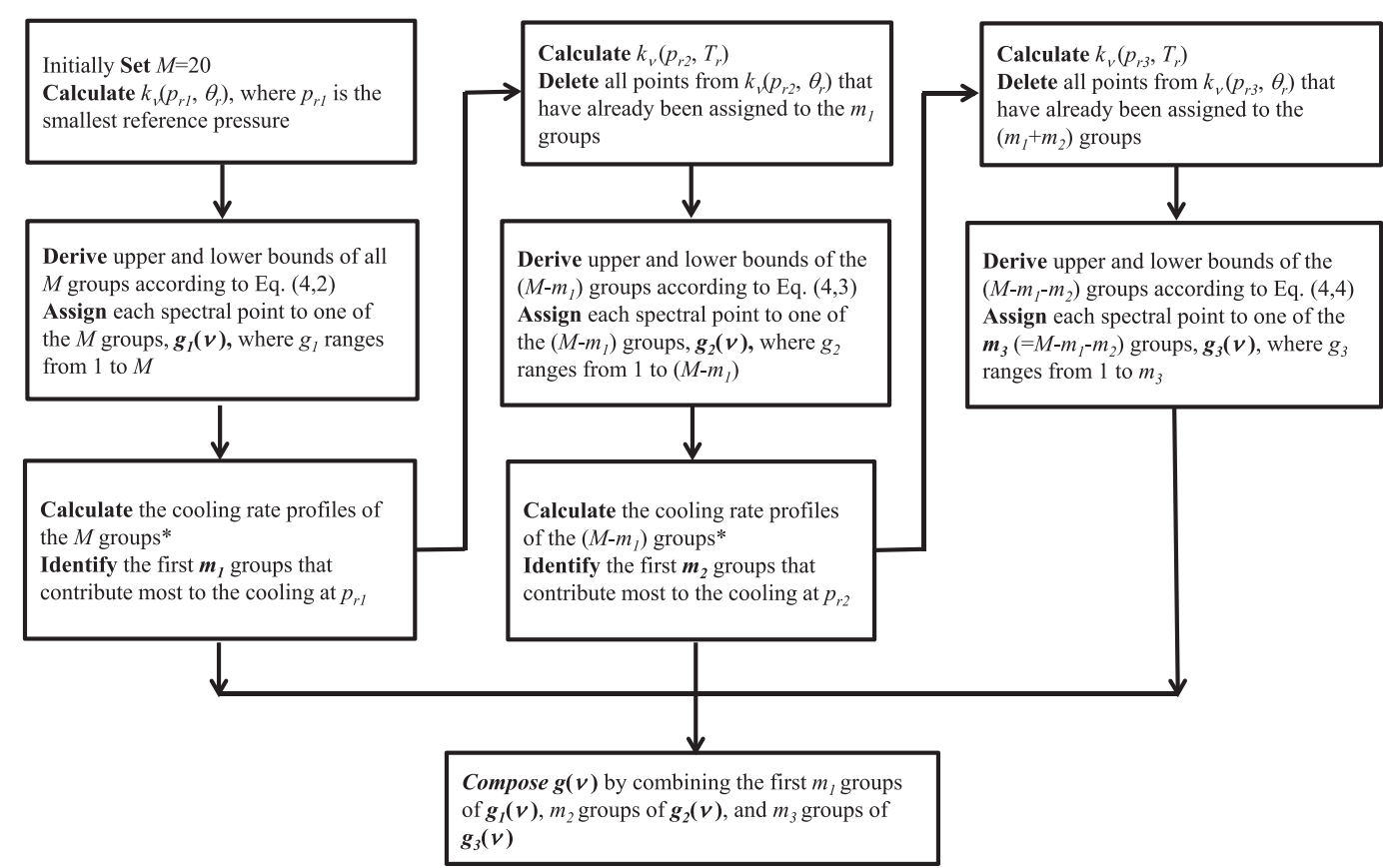

FIG. 2. Grouping of spectral points. This diagram is for the case of three reference pressures $p_{r 1}, p_{r 2}$, and $p_{r 3}$.

Because the $k_{g, \text { lin }}$ derived from Eq. (5.1) is larger than the $k_{g}$ derived from Eq. (3.3), emission of a layer, $I_{g}$, is overestimated when $k_{g}$ in Eq. (3.3) is replaced by $k_{g, \text { lin }}$. Thus, adjustment for $k_{g \text {,in }}$ to a smaller value is necessary unless either the magnitude or the range of $k_{\iota}$ in a $g$ group is small. Similar to Chou et al. (1998), a nonlinearly averaged $k_{g \text {,nonlin }}$ smaller than $k_{g, \text { lin }}$ is introduced, and the equivalent absorption coefficient is derived from

$$
\begin{aligned}
k_{g}(p, \theta)= & w_{g} k_{g, \text { lin }}(p, \theta)+\left(1-w_{g}\right) k_{g, \text { nonlin }}(p, \theta) \\
& 0<w_{g}<1 .
\end{aligned}
$$

To meet the requirement that $k_{g, \text { nonlin }}<k_{g, \text { lin }}$, the following nonlinear function is chosen:

$\log _{10} k_{g, \text { nonlin }}(p, \theta)=\frac{1}{B_{g}(\theta)} \sum_{i \in S_{g}} B_{i}(\theta) \log _{10} k_{i}(p, \theta) \delta \nu$

The weighting function $w_{g}$ approaches 1 for a small optical thickness and decreases with increasing optical thickness. It is empirically determined.

From Eqs. (3.24) and (5.2), the diffusivity-amplified equivalent absorption coefficient for the diffuse transmission function becomes

$$
\bar{k}_{g}(p, \theta)=\frac{1}{\mu_{g}}\left[w_{g} k_{g, \text { lin }}(p, \theta)+\left(1-w_{g}\right) k_{g, \text { nonlin }}(p, \theta)\right],
$$

where

$$
\mu_{g}=\cos \left(\varphi_{g}\right)
$$

The diffusivity factor $1 / \mu$ is close to 2 for weak absorption and decreases with increasing optical thickness. It is commonly set to 1.66 but is empirically determined as a function of $g$ in this study.

Derivations of the best fit of $w_{g}$ and $\mu_{g}$ are based on the LBL calculations of fluxes and the cooling rate of the typical tropical, midlatitude summer, and subarctic winter atmospheres. Because approximation for the nongray radiation of a layer by gray radiation would overestimate the absorption when layers are combined, minimization of errors in both fluxes and cooling rate is not feasible. Given the number of $g$ groups $M$, the best fit is the best choice of $w_{g}$ and $\mu_{g}$ that balances errors in both flux and cooling-rate calculations. Both $w_{g}$ and $\mu_{g}$ are functions of the absorber, spectral band, and $g$ group. The fitting, or adjustment, was conducted in two steps. In the first step, fitting for $w_{g}$ was carried out by computing the diffuse transmittance from Eq. (3.21) with $k_{g}$ given in Eq. (5.2). In the second step, by fixing the best fit of $w_{g}$, fitting for $\mu_{g}$ was carried out by computing the diffuse transmittance from Eq. (3.22) with $\bar{k}_{g}$ given in Eq. (5.4). It is noticed that the adjustment for $k_{g}$ or $\bar{k}_{g}$ is not feasible for individual $g$ groups in a correlated- $k$-distribution scheme as the spectral points in a given $g$ group are not the same throughout the entire column of the atmosphere, which is at odds with the LBL calculation. 
In certain bands, for example, the water vapor band 7 demonstrated in Fig. 1b, the absorption coefficient of a large portion of the spectral band is very small that the linear approximation for the transmission function, Eq. (5.1), applies and the weight $w_{g}$ in Eq. (5.2) can be set close to 1.0. Guided by the column-integrated water vapor amount of a humid tropical atmosphere, a threshold absorption coefficient $k_{\text {lin }}\left(p_{r}\right)$ that would satisfy Eq. (5.1) is specified. For the case of the water vapor band 7 , the best fit $w_{g}=0.9$ when setting $k_{\text {lin }}\left(p_{r}\right)=$ $10^{-24} \mathrm{~cm}^{2}$ molecule ${ }^{-1}$. This band uses only three $g$ groups, $M=3$. All the spectral points with $k_{\nu}\left(p_{r}\right)<$ $k_{\text {lin }}\left(p_{r}\right)$ are split to the third $g$ group. The Planckweighted $k$-distribution function $\mathrm{H}_{3}$ is 0.929 . Thus, this $g$ group comprises $92.9 \%$ of the "Planck weighted" spectral points, and only a small fraction, $7.1 \%$, is left for the first two $g$ groups. A small fraction of the total spectral points implies a small range of $k_{\nu}\left(p_{r}\right)$ and an accurate estimate for $k_{g}$, where $g=1,2$. This approach is applied to most of the bands for reducing the number $M$, including bands 4-8 and 11 of $\mathrm{H}_{2} \mathrm{O}$, bands 3, 5-7, and 11 of $\mathrm{CO}_{2}$, band 7 of $\mathrm{O}_{3}$, bands 3 and 9 of $\mathrm{N}_{2} \mathrm{O}$, and band 9 of $\mathrm{CH}_{4}$. Therefore, the efficiency of flux calculations is greatly enhanced. The best-fit number of $g$ groups for each of the 11 bands and 5 gases is given in Table 1.

Tables of $\bar{k}_{g}(p, \theta)$ given in Eq. (5.4) are precomputed using the LBL method with a spectral resolution of $0.001 \mathrm{~cm}^{-1}$. Each table corresponds to a given absorber, spectral band, and $g$ group. There are a total of 130 tables corresponding to the $130 \mathrm{~g}$ groups as shown in Table 1 . It is noticed that some $g$ groups involve only a single gas in this $k$-distribution scheme, whereas it might involve multiple gases in some correlated- $k$ distribution schemes. Therefore, the number of $g$ groups may not be an index for proper comparisons of the computational efficiency between this $k$-distribution scheme and other $k$-distribution or correlated- $k$-distribution schemes.

The size of a $\bar{k}_{g}(p, \theta)$ table is 52 pressures by 5 temperatures. The pressure ranges from 0.01 to $1059 \mathrm{hPa}$ with an interval of $\Delta \log _{10}(p)=0.1$. The temperature ranges by $100 \mathrm{~K}$ centered at a temperature profile taken from the mean of the tropical and subarctic winter atmospheres. The temperature interval $\Delta \theta$ is $25 \mathrm{~K}$. The temperature range of the $\bar{k}_{g}(p, \theta)$ table is $228-328 \mathrm{~K}$ at $1000 \mathrm{hPa}$ ( $\sim$ surface $), 157-257 \mathrm{~K}$ at $100 \mathrm{hPa}$ ( $\sim$ tropopause), $212-312 \mathrm{~K}$ at $0.6 \mathrm{hPa}(\sim$ stratopause), and $174-274 \mathrm{~K}$ at $0.01 \mathrm{hPa}$. In looking up $\bar{k}_{g}$ from the tables, it is bilinearly interpolated in $\log _{10}(p)$ and $\theta$.

\section{Sample flux and cooling-rate calculations}

The Planck-weighted $k$-distribution function $H_{g}$ and the diffusivity-amplified equivalent absorption coefficient $\bar{k}_{g}(p, \theta)$, are derived by empirically adjusting the parameters $M, J, m, w_{g}$, and $\mu_{g}$. The reason for adjusting $M$, as well as the associated $J$ and $m$, is to achieve a balance between the accuracy and speed of flux calculations, whereas the reason for adjusting $w_{g}$, and $\mu_{g}$ is to achieve a higher accuracy, as well as a balance between the accuracies in the cooling rate and the fluxes at the top of the atmosphere and the surface. Adjustments for these parameters are guided by imposing conditions on the accuracies of fluxes and cooling-rate calculations. In this study, the imposed conditions are 1) the coolingrate difference between the $k$-distribution and LBL calculations due to each gas in each spectral band is $<0.1 \mathrm{~K} \mathrm{day}^{-1}$ in the troposphere and $<1.0 \mathrm{~K} \mathrm{day}^{-1}$ in the stratosphere, and 2) the flux difference due to each gas in each spectral band is $<0.5 \mathrm{~W} \mathrm{~m}^{-2}$ at both the top of the atmosphere and the surface. These imposed conditions apply to all of the three typical atmospheres.

Table 2 demonstrates the maximum cooling-rate difference between the $k$-distribution and LBL calculations in the troposphere and the stratosphere due to the absorption by each of the five gases in each of the 11 bands. The maximum is taken over the layers in the respective sphere (troposphere or stratosphere) and over the three typical atmospheres. The difference is $<0.1 \mathrm{~K} \mathrm{day}^{-1}$ in the troposphere and $<0.7 \mathrm{~K} \mathrm{day}^{-1}$ in the stratosphere. Table 3 demonstrates the maximum difference in the outgoing longwave radiation at the top of the atmosphere, OLR, and the downward flux at the surface (SFC) due to the absorption by each of the five gases. The maximum is taken over the three typical atmospheres. The difference is $<0.50 \mathrm{~W} \mathrm{~m}^{-2}$ in all cases. Table 4 compares the total-band OLR and the downward flux at the surface due to absorption by each of the five gases in the three typical atmospheres. The tropical atmosphere has the largest differences of 0.68 and $2.00 \mathrm{~W} \mathrm{~m}^{-2}$, respectively, in the OLR and the downward flux at the surface. These accuracies are achieved with the use of the number of $g$ groups given in Table 1 .

Figure 3 demonstrates the total-band cooling rate and cooling-rate difference profiles due to the five gases in the midlatitude summer atmosphere. Water vapor is the most important absorber contributing to the tropospheric cooling, whereas $\mathrm{CO}_{2}$ is the most important in the stratosphere. The cooling due to $\mathrm{N}_{2} \mathrm{O}$ and $\mathrm{CH}_{4}$ is weak throughout the atmosphere. In the troposphere, the cooling-rate difference is $<0.1 \mathrm{~K} \mathrm{day}^{-1}$ for all of the five gases. In the stratosphere, the maximum coolingrate difference is $\sim 0.7 \mathrm{~K} \mathrm{day}^{-1}$, which is associated with the strong cooling due to $\mathrm{CO}_{2}$.

Figure 4 demonstrates the total-band cooling rate and cooling-rate difference due to overlapping absorption of 
TABLE 2. The maximum cooling-rate difference $\left(\mathrm{K} \mathrm{day}^{-1}\right)$ in the stratosphere and troposphere over the three typical atmospheres. The difference is the deviation of the $k$-distribution value from the line-by-line value, so it can be either positive or negative.

\begin{tabular}{|c|c|c|c|c|c|c|c|c|c|c|c|}
\hline & \multicolumn{11}{|c|}{ Spectral band } \\
\hline & 1 & 2 & 3 & 4 & 5 & 6 & 7 & 8 & 9 & 10 & 11 \\
\hline \multicolumn{12}{|c|}{$\mathrm{H}_{2} \mathrm{O}$} \\
\hline Stratosphere & 0.07 & 0.02 & 0.00 & 0.00 & 0.00 & 0.00 & 0.00 & 0.00 & 0.01 & 0.03 & 0.00 \\
\hline Troposphere & 0.06 & 0.08 & 0.05 & 0.05 & 0.02 & 0.05 & 0.02 & 0.02 & 0.03 & 0.03 & 0.01 \\
\hline \multicolumn{12}{|c|}{$\mathrm{CO}_{2}$} \\
\hline Troposphere & & & 0.01 & 0.02 & 0.01 & 0.00 & 0.00 & & & & 0.00 \\
\hline \multicolumn{12}{|c|}{$\mathrm{O}_{3}$} \\
\hline Stratosphere & & & & & & & 0.18 & & & & \\
\hline Troposphere & & & & & & & 0.01 & & & & \\
\hline \multicolumn{12}{|c|}{$\mathrm{N}_{2} \mathrm{O}$} \\
\hline Stratosphere & & & 0.00 & & & & 0.02 & & & & \\
\hline Troposphere & & & 0.00 & & & & 0.01 & & & & \\
\hline \multicolumn{12}{|c|}{$\mathrm{CH}_{4}$} \\
\hline Stratosphere & & & & & & & 0.04 & & & & \\
\hline Troposphere & & & & & & & 0.00 & & & & \\
\hline
\end{tabular}

the five gases for the three atmospheres. Generally, the cooling-rate difference is $<0.12 \mathrm{~K} \mathrm{day}^{-1}$ in the troposphere, and $<1.0 \mathrm{~K} \mathrm{day}^{-1}$ in the stratosphere. Table 5 demonstrates differences in the OLR and the downward flux at the surface for the same cases as Fig. 4. The maximum difference in the OLR is $<1.5 \mathrm{~W} \mathrm{~m}^{-2}$, which is only $0.5 \%$ of the OLR. The maximum difference in the downward surface flux is $1.6 \mathrm{~W} \mathrm{~m}^{-2}$, which is also a $0.5 \%$ difference.

In addition to the three typical atmospheres, two artificial atmospheres that mix the tropical atmosphere with the subarctic winter atmosphere are used for

TABLE 3. The maximum difference in the outgoing longwave radiation $\left(\mathrm{W} \mathrm{m}^{-2}\right)$ at the top of the atmosphere (OLR) and the downward flux at the surface (SFC) over the three typical atmospheres. The difference is the deviation of the $k$-distribution value from the line-byline value, so it can be either positive or negative.

\begin{tabular}{|c|c|c|c|c|c|c|c|c|c|c|c|}
\hline & \multicolumn{11}{|c|}{ Spectral band } \\
\hline & 1 & 2 & 3 & 4 & 5 & 6 & 7 & 8 & 9 & 10 & 11 \\
\hline \multicolumn{12}{|c|}{$\mathrm{H}_{2} \mathrm{O}$} \\
\hline OLR & 0.21 & 0.32 & 0.10 & 0.10 & 0.06 & 0.04 & 0.04 & 0.11 & 0.32 & 0.14 & 0.37 \\
\hline SFC & 0.00 & 0.37 & 0.41 & 0.36 & 0.23 & 0.47 & 0.08 & 0.14 & 0.13 & 0.04 & 0.52 \\
\hline \multicolumn{12}{|c|}{$\mathrm{CO}_{2}$} \\
\hline OLR & & & 0.12 & 0.42 & 0.37 & 0.04 & 0.04 & & & & 0.10 \\
\hline SFC & & & 0.24 & 0.10 & 0.49 & 0.07 & 0.05 & & & & 0.17 \\
\hline \multicolumn{12}{|c|}{$\mathrm{O}_{3}$} \\
\hline OLR & & & & & & & 0.40 & & & & \\
\hline SFC & & & & & & & 0.14 & & & & \\
\hline \multicolumn{12}{|c|}{$\mathrm{N}_{2} \mathrm{O}$} \\
\hline OLR & & & 0.06 & & & & & & 0.22 & & \\
\hline SFC & & & 0.02 & & & & & & 0.05 & & \\
\hline \multicolumn{12}{|c|}{$\mathrm{CH}_{4}$} \\
\hline OLR & & & & & & & & & 0.03 & & \\
\hline SFC & & & & & & & & & 0.27 & & \\
\hline
\end{tabular}


TABLE 4. Comparison of the line-by-line (LBL)- and the $k$-distribution (KD)-calculated total-band outgoing longwave radiation (OLR; $\mathrm{W} \mathrm{m}^{-2}$ ) and the downward flux at the surface (SFC; $\mathrm{W} \mathrm{m}^{-2}$ ) due to absorption by each of the five gases. The spectral bands included for each gas are given in Table 1. TRP, MLS, and SAW denote, respectively, the typical tropical, midlatitude summer, and subarctic winter atmospheres.

\begin{tabular}{llrrrrr}
\hline \hline & & \multicolumn{2}{c}{ OLR } & & \multicolumn{2}{c}{ SFC } \\
\cline { 3 - 4 } \cline { 6 - 7 } & & LBL & KD - LBL & & LBL & KD - LBL \\
\hline $\mathrm{H}_{2} \mathrm{O}$ & TRP & 351.98 & -0.36 & & 305.47 & 2.00 \\
& MLS & 335.19 & -0.09 & & 272.69 & 0.65 \\
& SAW & 224.58 & -0.07 & & 127.40 & -1.19 \\
$\mathrm{CO}_{2}$ & TRP & 182.34 & -0.68 & & 85.22 & 0.67 \\
& MLS & 170.96 & -0.51 & & 79.16 & 0.69 \\
& SAW & 100.58 & -0.25 & & 46.00 & 0.57 \\
$\mathrm{O}_{3}$ & TRP & 27.01 & -0.25 & & 3.57 & -0.14 \\
& MLS & 23.05 & -0.40 & & 4.35 & -0.06 \\
& SAW & 11.00 & -0.29 & & 2.80 & 0.08 \\
$\mathrm{~N}_{2} \mathrm{O}$ & TRP & 60.61 & -0.17 & & 6.95 & -0.07 \\
& MLS & 55.89 & -0.13 & & 6.49 & -0.04 \\
& SAW & 31.71 & -0.03 & & 3.68 & 0.04 \\
$\mathrm{CH}_{4}$ & TRP & 21.61 & 0.03 & & 6.75 & -0.27 \\
& MLS & 19.27 & 0.03 & & 6.12 & -0.23 \\
& SAW & 8.19 & -0.01 & & 2.77 & -0.06 \\
\hline
\end{tabular}

independent validation of the $k$-distribution scheme. One has the subarctic winter temperature and tropical water vapor, SAW-T: TRP-W, and the other has the tropical temperature and subarctic winter water vapor, TRP-T: SAW-W. These two artificial atmospheres differ by a factor of $\sim 10$ in the column-integrated water vapor $\left(0.34 \mathrm{vs} 4.0 \mathrm{~g} \mathrm{~cm}^{-2}\right)$ and by $43 \mathrm{~K}$ in the surface temperature $(257$ vs $300 \mathrm{~K})$. These two extreme atmospheres should be able to enclose the temperature and humidity variabilities in most of the real atmospheres.

Figure 5 demonstrates the profiles of the water vapor cooling rate and cooling-rate difference between the $k$ distribution (KD) and LBL of the two artificial atmospheres. Similar to the midlatitude summer case demonstrated in Fig. 3, the cooling-rate difference is $<0.1 \mathrm{~K} \mathrm{day}^{-1}$ throughout the entire column of the atmosphere, except near $0.01 \mathrm{hPa}$ where the difference is $<0.3 \mathrm{~K} \mathrm{day}^{-1}$. Table 6 demonstrates the difference in the OLR and the downward surface flux between the KD and LBL in each and total spectral bands. The difference is generally small, $<1.0 \mathrm{~W} \mathrm{~m}^{-2}$, except for the TRP-T: SAW-W case that the difference in the total-band surface downward flux is $2.93 \mathrm{~W} \mathrm{~m}^{-2}$.

For the application to future scenarios, we tested the $k$-distribution scheme by doubling the concentrations of $\mathrm{CO}_{2}, \mathrm{~N}_{2} \mathrm{O}$, and $\mathrm{CH}_{4}$ to $800,0.64$, and 3.60 ppmv, respectively. Overlapping of gas absorption is included.
Figure 6 demonstrates the cooling rate and the coolingrate difference between the KD and LBL for a doubled $\mathrm{CO}_{2}, \mathrm{~N}_{2} \mathrm{O}$, and $\mathrm{CH}_{4}$, respectively, in the midlatitude summer atmosphere. Similar to the normal $\mathrm{CO}_{2} \mathrm{dem}-$ onstrated in Fig. 3b, the difference for the doubled $\mathrm{CO}_{2}$ demonstrated in Fig. $6 \mathrm{a}$ is $<0.1 \mathrm{Kday}^{-1}$ in the troposphere and $<1.0 \mathrm{~K} \mathrm{day}^{-1}$ in the stratosphere near $0.01 \mathrm{hPa}$. The cooling-rate difference for a double $\mathrm{N}_{2} \mathrm{O}$, and $\mathrm{CH}_{4}$ as demonstrated in Figs. $6 \mathrm{~b}$ and $6 \mathrm{c}$ is very small, $<0.05 \mathrm{~K} \mathrm{day}^{-1}$ throughout the troposphere and stratosphere. Table 7 demonstrates that the difference in the impact of a doubled greenhouse gas concentration on the OLR between the KD and LBL is $<0.3 \mathrm{~W} \mathrm{~m}^{-2}$ for all bands and gases.

For independent validation of the $k$-distribution approximation scheme, we further sampled eight atmospheres in four diversified climatic regimes and two seasons (June and December). Table 8 demonstrates the four sites where the sample atmospheres are located and the time of the samples. Those samples include the atmospheres in the Atmospheric Radiation Measurement (ARM) Southern Great Plains in June (SGP-J) and December (SGP-D), ARM north slope of Alaska in June (NSA-J) and December (NSA-D), warm pool western tropical Pacific in June (WTP-J) and December (WTP-D), and cold pool eastern tropical Pacific in June (ETP-J) and December (ETP-D). Clear-sky temperature and humidity of these eight atmospheres were randomly sampled from the ECMWF ERA5 reanalysis (https://www.ecmwf.int/en/forecasts/datasets/reanalysisdatasets/era5). The ECMWF ERA5 temperature and humidity available to us are defined at 37 pressure levels ranging from 1 to $1000 \mathrm{hPa}$. Table 8 demonstrates that these samples range from highly cold and dry atmosphere to warm and humid atmosphere with the skin temperature differs by $54 \mathrm{~K}$ and the column-integrated water vapor amount by a factor of 25 . The ozone data are taken from the U.S. NASA Goddard Aura Ozone Monitoring Instrument level-2 Ozone Profile data product OMO3PR (Haan and Veefkind 2009). The OMO3PR product provides ozone values (in Dobson units, DU) for 18 atmospheric layers. We interpolated the ozone amount (DU) in the 18 layers to the mean ozone mixing ratio at the same 37 pressure levels as that of temperature and humidity. Also demonstrated in Table 8 are the column-integrated ozone amount of each of the sample atmospheres. It ranges by a factor of 1.7 from 245 DU in the western tropical Pacific in December to 417 DU on the northern slope of Alaska in December.

The concentrations of $\mathrm{N}_{2} \mathrm{O}$ and $\mathrm{CH}_{4}$ have been demonstrated to decrease with height in the stratosphere (e.g., Seinfeld and Pandis 2006; Tsai et al. 2012; Zhou et al. 2019). To test the validity of the $k$-distribution scheme in 

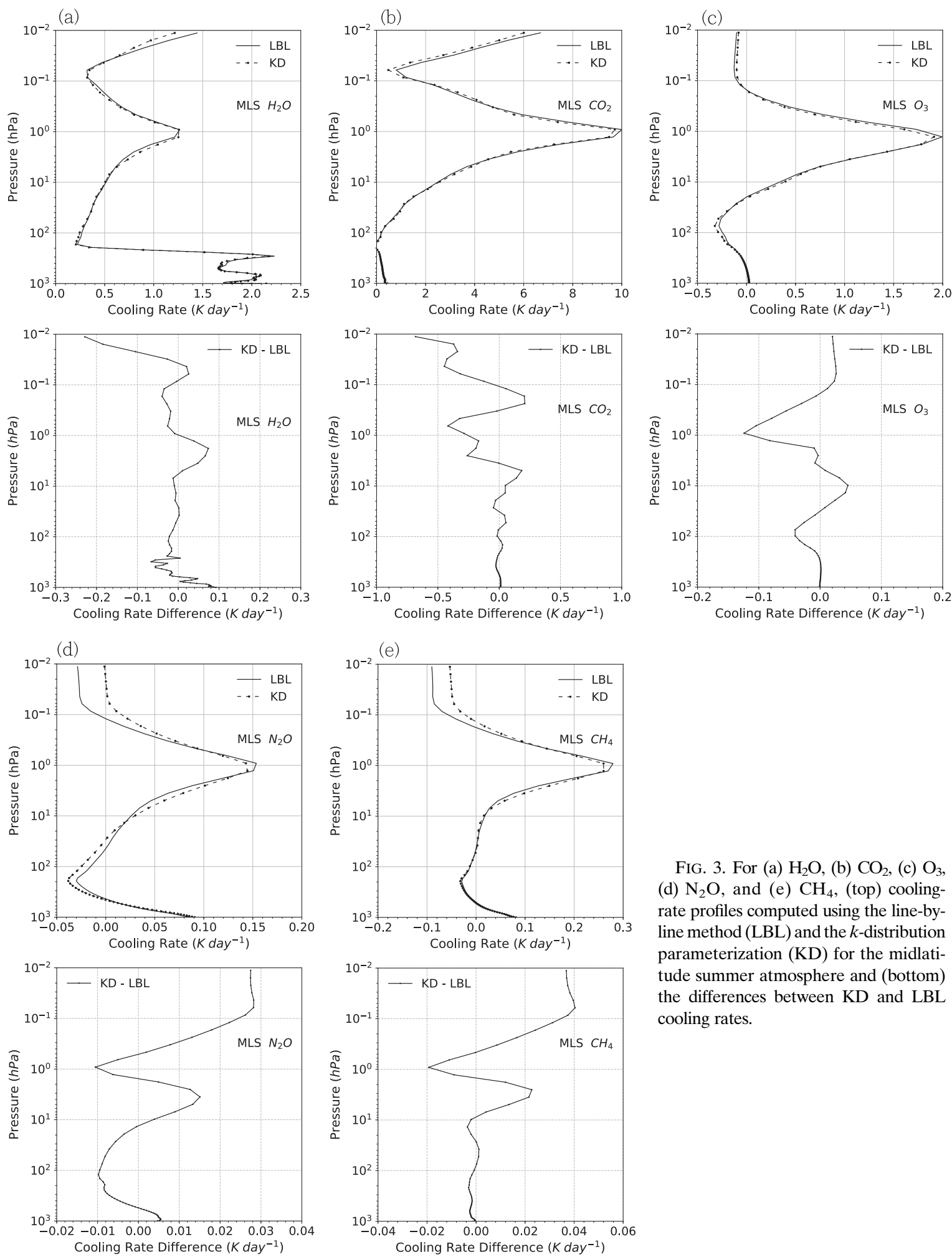

FIG. 3. For (a) $\mathrm{H}_{2} \mathrm{O}$, (b) $\mathrm{CO}_{2}$, (c) $\mathrm{O}_{3}$, (d) $\mathrm{N}_{2} \mathrm{O}$, and (e) $\mathrm{CH}_{4}$, (top) coolingrate profiles computed using the line-byline method (LBL) and the $k$-distribution parameterization (KD) for the midlatitude summer atmosphere and (bottom) the differences between KD and LBL cooling rates. 
(a)
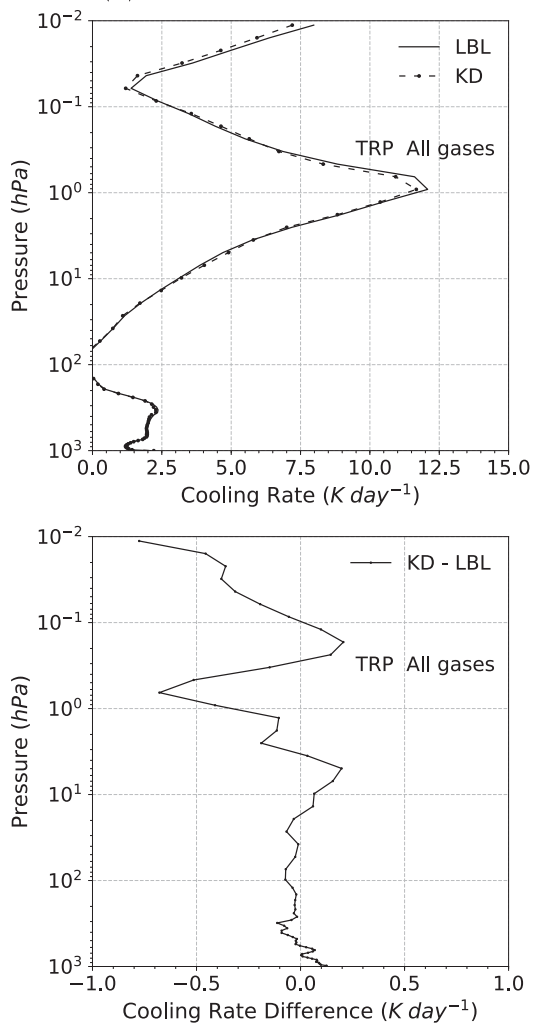

(b)
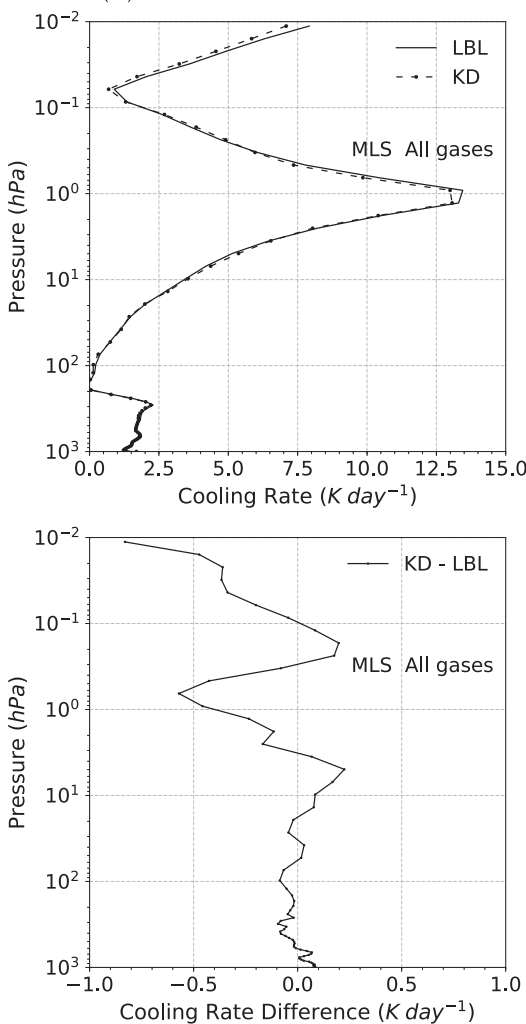

(c)
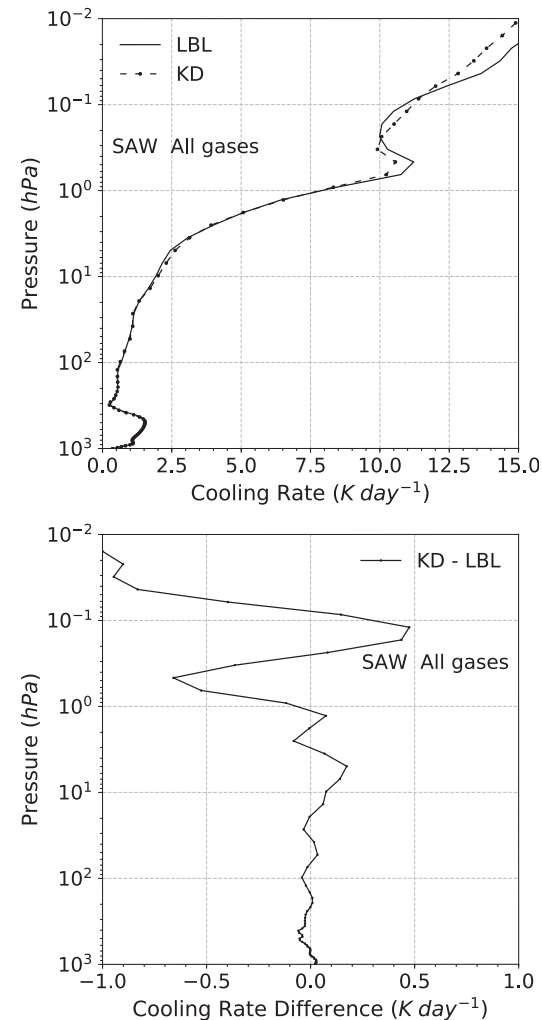

FIG. 4. For (a) midlatitude summer (MLS), (b) tropical (TRP), and (c) subarctic winter (SAW) atmospheres, (top) cooling-rate profiles with overlapping absorption of the five gases and (bottom) the differences between KD and LBL cooling rates.

the situation that the stratospheric concentrations of $\mathrm{N}_{2} \mathrm{O}$ and $\mathrm{CH}_{4}$ decrease with height, we set the following vertical profiles: (i) the $\mathrm{N}_{2} \mathrm{O}$ and $\mathrm{CH}_{4}$ concentrations are set, respectively to 0.32 and $1.80 \mathrm{ppmv}$ below the $100 \mathrm{hPa}$ pressure level; (ii) between 10 and $100 \mathrm{hPa}$, the concentrations decrease linearly with the logarithm of pressure. Specifically, the concentrations at $10 \mathrm{hPa}$ are set to be $1 / 3$ of the values at $100 \mathrm{hPa}$; and (iii) above the $10 \mathrm{hPa}$ pressure level, the concentrations are again set to be constant as the values at $10 \mathrm{hPa}$. In flux and cooling-rate calculations, we artificially added a layer above $1 \mathrm{hPa}$, and set the temperature and gas concentration to the values at $1 \mathrm{hPa}$.

Figure 7 demonstrates the cooling-rate profiles from LBL and KD calculations and the difference between them for each of the five absorbing gases. For a given absorbing gas, we selected from the eight sample atmospheres the one with the least satisfactory KDcalculated cooling-rate profile when compared with the LBL calculations. For water vapor (Fig. 7a), the SGP-J has the least satisfactory KD cooling profile among the eight sample atmospheres. The temperature and humidity profiles of SGP-J are shown in Fig. 8. SGP-J is hot and humid with a skin temperature of $302.7 \mathrm{~K}$.
The column-integrated water vapor amount is $42.3 \mathrm{~kg} \mathrm{~m}^{-2}$, which is nearly twice as much as the MLS atmosphere. There is a strong humidity inversion in the layer 300$400 \mathrm{hPa}$. The maximum cooling rate of $5 \mathrm{~K} \mathrm{day}^{-1}$ at $800 \mathrm{hPa}$ is nearly twice as large as the maximum cooling of $\sim 2.2 \mathrm{~K} \mathrm{day}^{-1}$ in the MLS atmosphere as demonstrated in Fig. 3a. The maximum difference between the $\mathrm{KD}$ and $\mathrm{LBL}$ is $\sim 0.2 \mathrm{~K} \mathrm{day}^{-1}$, which is also nearly twice as large as that of the MLS atmosphere. Figure $7 \mathrm{~b}$ demonstrates the least satisfactory $\mathrm{KD} \mathrm{CO}_{2}$ cooling profile of NSA-J. The relatively large difference between KD and LBL in the upper stratosphere is associated with the large cooling rate, which is in turn associated the high stratospheric temperature as shown in Fig. 8. For $\mathrm{O}_{3}, \mathrm{~N}_{2} \mathrm{O}$, and $\mathrm{CH}_{4}$, the cooling-rate

TABLE 5. As in Table 4, but for the overlapping absorption of all five gases.

\begin{tabular}{lccccc}
\hline & \multicolumn{2}{c}{ OLR } & & \multicolumn{2}{c}{ SFC } \\
\cline { 2 - 3 } \cline { 5 - 6 } & LBL & KD - LBL & & LBL & KD - LBL \\
\hline TRP & 303.40 & -1.33 & & 337.24 & 1.58 \\
MLS & 290.39 & -1.13 & & 308.14 & 0.77 \\
SAW & 201.06 & -0.73 & & 166.03 & -0.45 \\
\hline
\end{tabular}


(a)
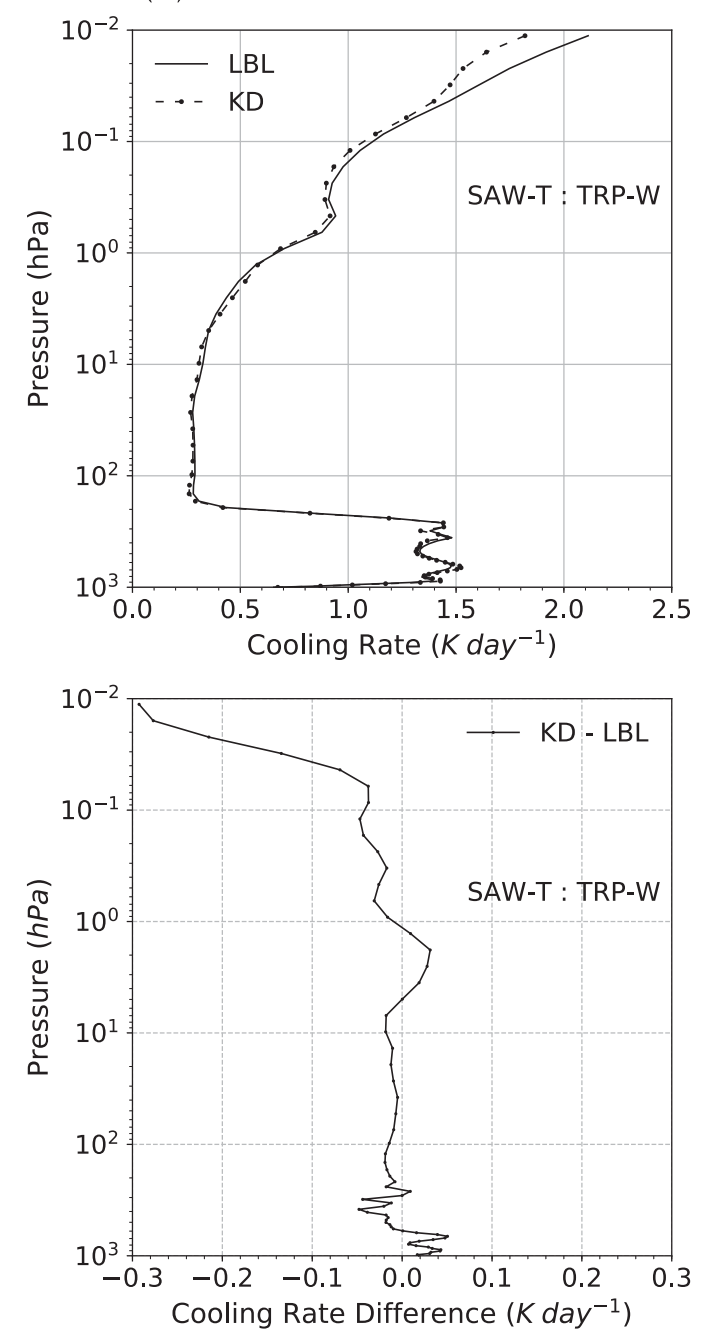

(b)
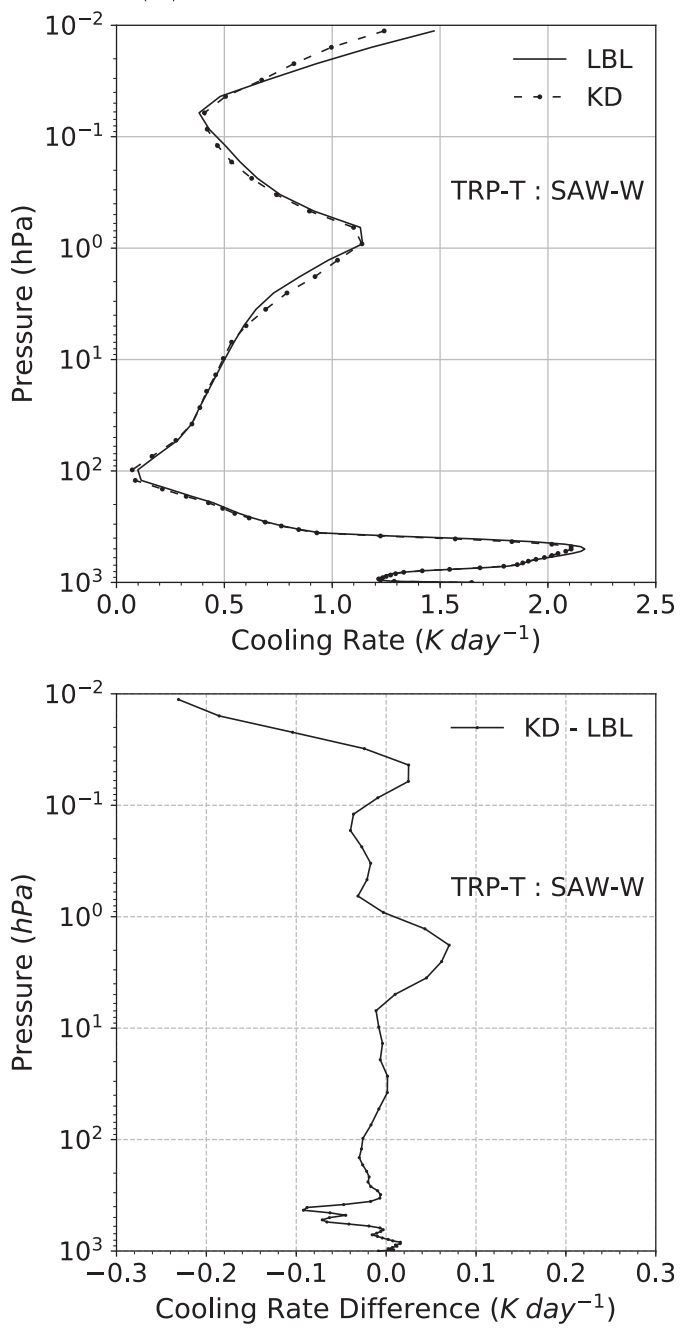

FIG. 5. For (a) SAW-T: TRP-W atmosphere, which has the SAW temperature and TRP humidity, and (b) TRP-T: SAW-W atmosphere, which has TRP temperature and SAW humidity, (top) water vapor cooling-rate profiles computed using KD and LBL methods and (bottom) differences between the KD and LBL cooling rates.

differences demonstrated in Fig. 7 are all small; $<0.08 \mathrm{~K} \mathrm{day}^{-1}$ for $\mathrm{O}_{3}$, and $<0.02 \mathrm{~K} \mathrm{day}^{-1}$ for $\mathrm{N}_{2} \mathrm{O}$ and $\mathrm{CH}_{4}$. Fluxes and cooling rate due to $\mathrm{N}_{2} \mathrm{O}$ and $\mathrm{CH}_{4}$ were also calculated for the cases that the $\mathrm{N}_{2} \mathrm{O}$ and $\mathrm{CH}_{4}$ concentrations are constants throughout the atmosphere. The results (not shown in the figures and tables) are nearly identical to that shown in Table 9 for fluxes and similar to Figs. $7 \mathrm{~d}$ and $7 \mathrm{e}$ for the cooling rate.

Table 9 demonstrates the mean, root-mean-square (RMS), and maximum of the difference between the KD and LBL calculations of OLR and the downward surface radiation of the eight sample atmospheres. The maximum difference is the largest deviation of KD from LBL among those 8 atmospheres. So, it can be either positive or negative. The maximum deviations of OLR and the downward flux at the surface are all $<1 \mathrm{~W} \mathrm{~m}^{-2}$ for all sample atmospheres, except for GSP-J, which has a maximum difference of $2.68 \mathrm{~W} \mathrm{~m}^{-2}$ in the downward surface flux due to water vapor. The maximum differences are all small, $<0.53 \mathrm{~W} \mathrm{~m}^{-2}$ for $\mathrm{O}_{3}, \mathrm{~N}_{2} \mathrm{O}$, and $\mathrm{CH}_{4}$. We also calculated fluxes and cooling rate for cases that the $\mathrm{N}_{2} \mathrm{O}$ and $\mathrm{CH}_{4}$ concentrations did not change with height. The results (not shown in tables and figures) are nearly identical to that given in Table 9 in fluxes and similar to Figs. $7 \mathrm{~d}$ and $7 \mathrm{e}$ in cooling rate.

\section{Summary and concluding remarks}

A new $k$-distribution scheme without the correlation assumption is developed based on LBL calculations of 
TABLE 6. Differences between the KD and LBL calculations $(\mathrm{KD}-\mathrm{LBL})$ in the OLR $\left(\mathrm{W} \mathrm{m}^{-2}\right)$ and the downward flux at the surface $\left(\mathrm{SFC} ; \mathrm{W} \mathrm{m}^{-2}\right.$ ) in each and total spectral bands. SAW-T: TRP-W denotes the atmosphere with SAW temperature and TRP humidity, whereas TRP-T: SAW-W denotes the atmosphere with TRP temperature and SAW humidity.

\begin{tabular}{|c|c|c|c|c|c|c|c|c|c|c|c|c|}
\hline \multicolumn{13}{|c|}{ Spectral band } \\
\hline & 1 & 2 & 3 & 4 & 5 & 6 & 7 & 8 & 9 & 10 & 11 & Total \\
\hline \multicolumn{13}{|c|}{ SAW-T: TRP-W } \\
\hline OLR & -0.12 & -0.12 & 0.09 & 0.04 & 0.03 & 0.02 & 0.01 & 0.00 & 0.04 & -0.04 & -0.01 & -0.05 \\
\hline SFC & 0.00 & 0.23 & 0.29 & -0.30 & -0.02 & -0.12 & -0.04 & -0.05 & 0.00 & 0.00 & 0.04 & 0.04 \\
\hline \multicolumn{13}{|c|}{ TRP-T: SAW-W } \\
\hline OLR & -0.27 & -0.21 & 0.24 & 0.14 & 0.10 & 0.07 & 0.04 & 0.11 & 0.44 & -0.11 & -0.20 & 0.36 \\
\hline $\mathrm{SFC}$ & 0.01 & 0.22 & -0.72 & -0.95 & -0.34 & -0.32 & -0.13 & -0.28 & -0.72 & 0.10 & 0.20 & -2.93 \\
\hline
\end{tabular}

the absorption coefficient using the 2012 edition of the High-Resolution Transmission (HITRAN) molecular line parameters. All spectral points in a spectral band are split to a number of groups, or $g$ groups. A $g$ group is then treated as monochromatic in flux calculations. Grouping of the spectral points are based on a few sets of reference pressure and temperature $\left(p_{r}, \theta_{r}\right)$ corresponding to the heights where cooling rate is most prominent. In this new $k$-distribution scheme, the absorption coefficients at the reference pressures and temperatures $k_{\nu}\left(p_{r}, \theta_{r}\right)$ of a spectral band are divided into a total of $M$ equal intervals in $\log _{10}\left[k_{\nu}\left(p_{r}, \theta_{r}\right)\right]$. A spectral point $\nu$ is identified with one of the $M$ intervals, or $g$ groups, according to $k_{\nu}\left(p_{r}, \theta_{r}\right)$. This defines the $g$ group where a spectral point belongs. Different from the correlated- $k$ distribution scheme, a spectral point can only belong to the same $g$ group in computing radiative transfer through an inhomogeneous path in the atmosphere. Associated with a $g$ group are the equivalent absorption coefficient $k_{g}$ and the Planck-weighted $k$-distribution (a)
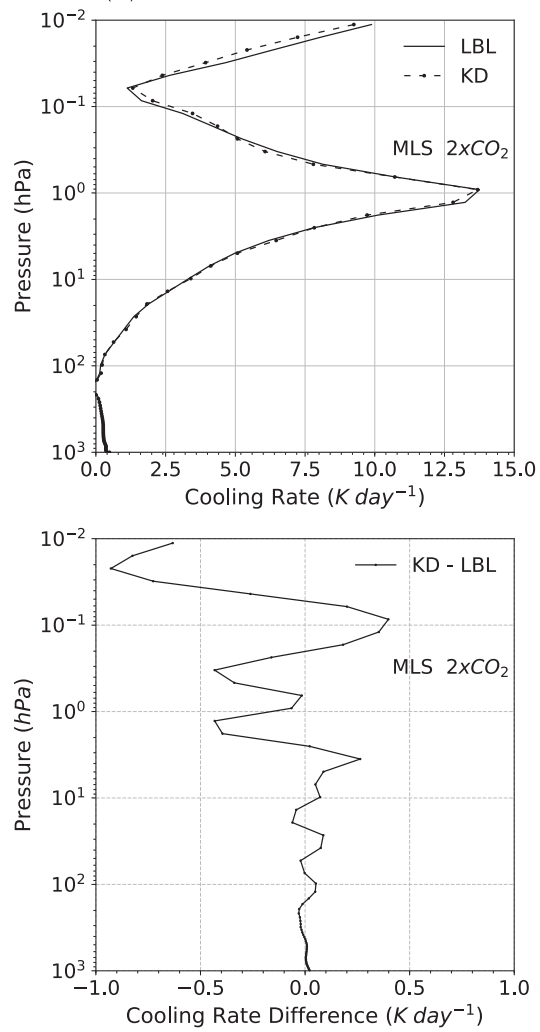

(b)
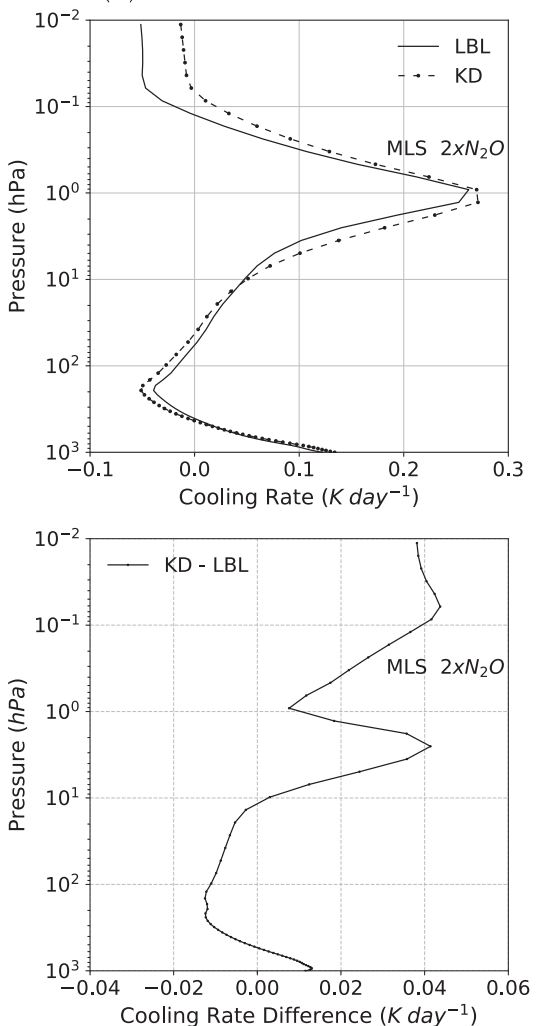

(c)
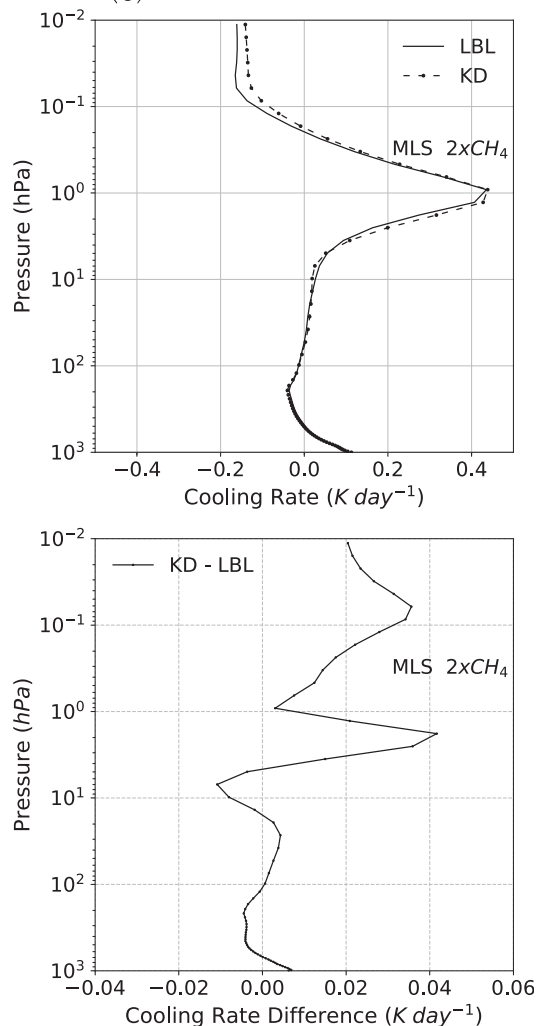

FIG. 6. For the midlatitude summer atmosphere of (a) doubled $\mathrm{CO}_{2}$, (b) doubled $\mathrm{N}_{2} \mathrm{O}$, and (c) doubled $\mathrm{CH}_{4}$, (top) cooling-rate profiles computed using KD and LBL methods and (bottom) differences between the KD and LBL cooling rates. 
TABLE 7. Changes in the outgoing longwave radiation (OLR; $\mathrm{W} \mathrm{m}^{-2}$ ) due to a doubling of the $\mathrm{CO}_{2}, \mathrm{~N}_{2} \mathrm{O}$, and $\mathrm{CH}_{4}$ concentrations in the midlatitude summer atmosphere. Overlapping of absorption is included for those gases and bands given in Table 1 . KD - LBL denotes the difference between KD and LBL.

\begin{tabular}{|c|c|c|c|c|c|c|c|c|c|c|}
\hline \multirow[b]{2}{*}{ Spectral band } & \multirow{2}{*}{$\begin{array}{l}\text { Spectral range } \\
\quad\left(\mathrm{cm}^{-1}\right)\end{array}$} & \multicolumn{3}{|c|}{$\mathrm{CO}_{2}$} & \multicolumn{3}{|c|}{$\mathrm{N}_{2} \mathrm{O}$} & \multicolumn{3}{|c|}{$\mathrm{CH}_{4}$} \\
\hline & & LBL & KD & $\mathrm{KD}-\mathrm{LBL}$ & LBL & KD & $\mathrm{KD}-\mathrm{LBL}$ & LBL & KD & $\mathrm{KD}-\mathrm{LBL}$ \\
\hline 3 & $540-620$ & -1.13 & -1.24 & -0.11 & -0.25 & -0.33 & -0.08 & & & \\
\hline 4 & $620-720$ & 0.10 & 0.30 & 0.21 & & & & & & \\
\hline 5 & $720-800$ & -1.56 & -1.71 & -0.15 & & & & & & \\
\hline 6 & $800-980$ & -0.31 & -0.32 & -0.01 & & & & & & \\
\hline 7 & $980-110$ & -0.20 & -0.22 & -0.02 & & & & & & \\
\hline 9 & $1215-1380$ & & & & -0.50 & -0.70 & -0.20 & & & \\
\hline 11 & $1900-3000$ & -0.11 & -0.11 & 0.00 & & & & -1.53 & -1.57 & -0.04 \\
\hline Total & & -3.21 & -3.30 & -0.09 & -0.75 & -1.03 & -0.28 & -1.53 & -1.57 & -0.04 \\
\hline
\end{tabular}

function $H_{g}$. A linear average of the absorption coefficients in a $g$ group would always overestimate the absorption. In this study, the $k_{g}$ is derived by taking the weighted mean of the linear average and the nonlinear average of the absorption coefficients. The weight is empirically determined as a function of $g$ group because it is dependent upon the optical thickness and, hence, the absorber amount. The $k_{g}$ is further amplified by a diffusivity factor, $1 / \mu_{g}$, to approximate the angular integration for the diffuse transmission function. The diffusivity factor is commonly set to 1.66 , but it is also empirically determined as a function of $g$ group in this study.

The longwave spectrum between 20 and $3000 \mathrm{~cm}^{-1}$ is divided into 11 bands, and the $k$-distribution scheme is applied to $\mathrm{H}_{2} \mathrm{O}, \mathrm{CO}_{2}, \mathrm{O}_{3}, \mathrm{~N}_{2} \mathrm{O}$, and $\mathrm{CH}_{4}$. Fluxes and cooling rate are calculated using the $k$-distribution scheme and results compared with LBL calculations for three typical tropical, midlatitude summer, and subarctic winter atmospheres, as well as two extreme artificial atmospheres. For individual gases, the cooling-rate difference is $<0.2 \mathrm{~K} \mathrm{day}^{-1}$ in the troposphere, and $<1.0 \mathrm{~K} \mathrm{day}^{-1}$ below the $0.01 \mathrm{hPa}$ level in the stratosphere. The flux difference is $<0.7 \mathrm{~W} \mathrm{~m}^{-2}$ at the top of the atmosphere, and $<3.0 \mathrm{~W} \mathrm{~m}^{-2}$ at the surface.
For the cases with overlapping absorption of all five gases, the total-band cooling-rate difference is $<0.12 \mathrm{~K} \mathrm{day}^{-1}$ in the troposphere, and $<1.0 \mathrm{Kday}^{-1}$ in the stratosphere below the $0.01 \mathrm{hPa}$ level. The flux difference is $<1.5 \mathrm{~W} \mathrm{~m}^{-2}$ at the top of the atmosphere, and $<1.6 \mathrm{~W} \mathrm{~m}^{-2}$ at the surface. These differences are attained with the use of $130 \mathrm{~g}$ groups, which is the sum of the $g$ groups of all five gases. For independent validation of the $k$-distribution approximation scheme, we further chose eight clear-sky atmospheres in four diversified climatic regimes and two seasons sampled from ERA5. Flux and cooling-rate differences between the KD and LBL calculations are comparable to the cases of the three typical atmospheres and two extreme artificial atmospheres.

The approach of this $k$-distribution scheme to empirically deriving the diffusivity-amplified equivalent absorption coefficient $\bar{k}_{g}$ for diffuse transmittance is not feasible in a correlated- $k$ distribution scheme. For a correlated- $k$ distribution approximation, the spectral points of a given $g$ group at various pressure and temperature are not identical, but they are assumed identical; hence the correlated- $k$ assumption. Because the spectral points in a $g$ group are not identical along a path in the atmosphere, fluxes and cooling rate cannot be

TABLE 8. Locations, time, skin temperatures, and column-integrated water vapor and ozone amounts of the eight sample atmospheres used for independent validation of the $k$-distribution approximation.

\begin{tabular}{|c|c|c|c|c|c|c|}
\hline Location-month & $\begin{array}{l}\text { Latitude } \\
\quad\left({ }^{\circ}\right)\end{array}$ & $\begin{array}{l}\text { Longitude } \\
\qquad\left(^{\circ}\right)\end{array}$ & $\begin{array}{c}\text { Time } \\
\text { (YYYYMMDDHH) }\end{array}$ & $\begin{array}{c}\text { Skin } \\
\text { temperature } \\
(\mathrm{K})\end{array}$ & $\begin{array}{c}\text { Column } \\
\mathrm{H}_{2} \mathrm{O} \\
\left(\mathrm{kg} \mathrm{m}^{-2}\right)\end{array}$ & $\begin{array}{l}\text { Column } \mathrm{O}_{3} \\
\text { (DU) }\end{array}$ \\
\hline Southern Great Plains (SGP)-June & 36.605 & 263.485 & 2018060114 & 302.72 & 42.30 & 323 \\
\hline Southern Great Plains (SGP)-December & 36.605 & 263.485 & 2018120201 & 281.16 & 11.86 & 314 \\
\hline North slope of Alaska (NSA)-June & 71.323 & 156.609 & 2018061107 & 274.73 & 9.95 & 329 \\
\hline North slope of Alaska (NSA)-December & 71.323 & 156.609 & 2014121105 & 248.18 & 1.82 & 417 \\
\hline Western tropical Pacific (WTP)-June & 10.000 & 140.000 & 2017060823 & 302.62 & 44.78 & 265 \\
\hline Western tropical Pacific (WTP)-December & 10.000 & 140.000 & 2013121011 & 301.83 & 37.40 & 245 \\
\hline Eastern tropical Pacific (ETP)-June & -10.000 & 260.000 & 2016060323 & 299.70 & 22.87 & 246 \\
\hline Eastern tropical Pacific (ETP)-December & -10.000 & 260.000 & 2018122822 & 295.96 & 22.45 & 257 \\
\hline
\end{tabular}


(a)
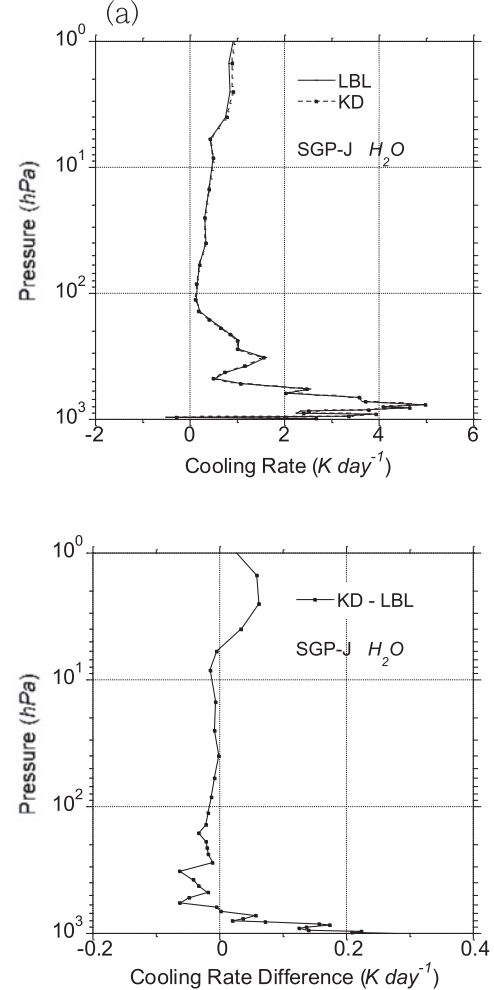

(d)
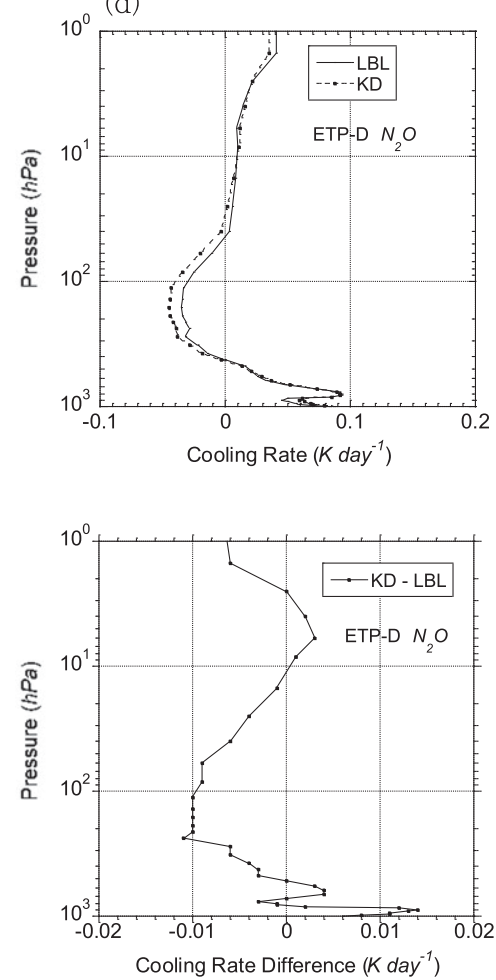

(b)
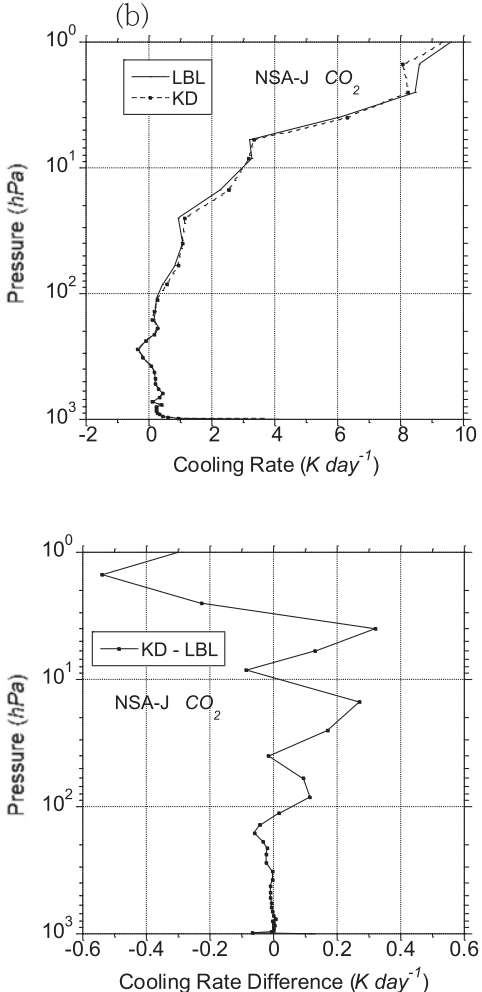

(e)
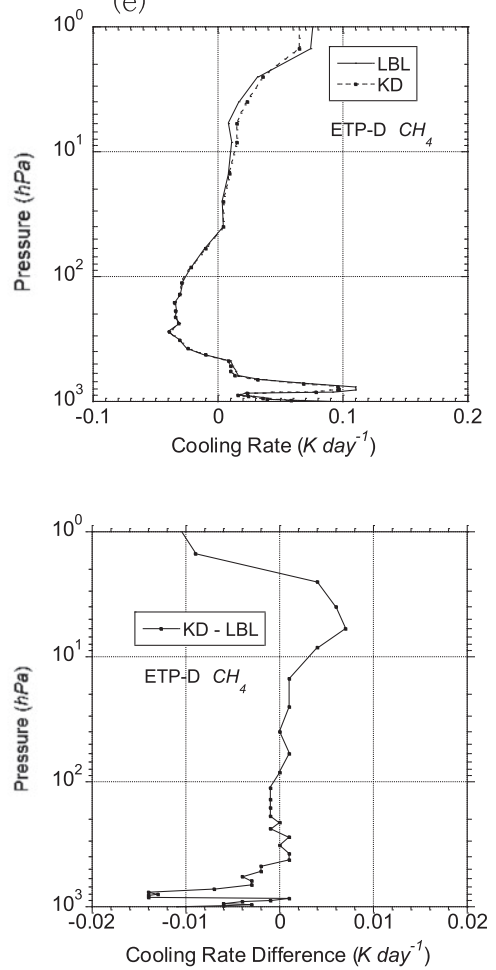
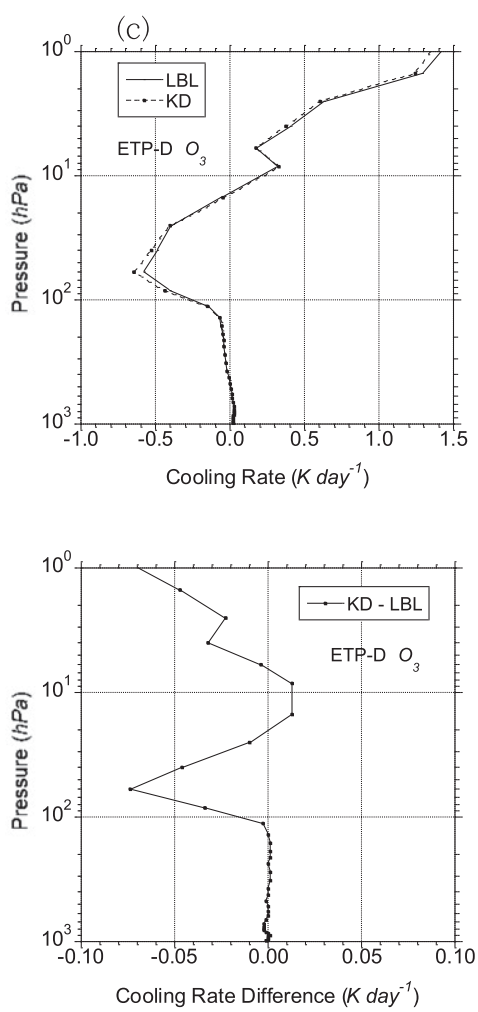

FIG. 7. For (a) $\mathrm{H}_{2} \mathrm{O}$ in SGP-J, (b) $\mathrm{CO}_{2}$ in NSA-J, (c) $\mathrm{O}_{3}$ in ETP-D, (d) $\mathrm{N}_{2} \mathrm{O}$ in ETP-D, and (e) $\mathrm{CH}_{4}$ in ETP-D, (top) cooling-rate profiles computed using KD and LBL and (bottom) differences between the KD and LBL cooling rates. 
(a)

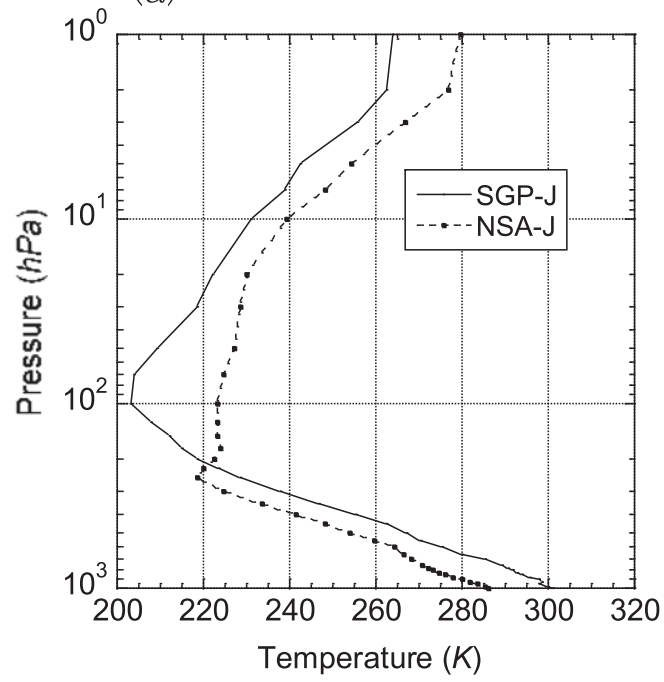

(b)

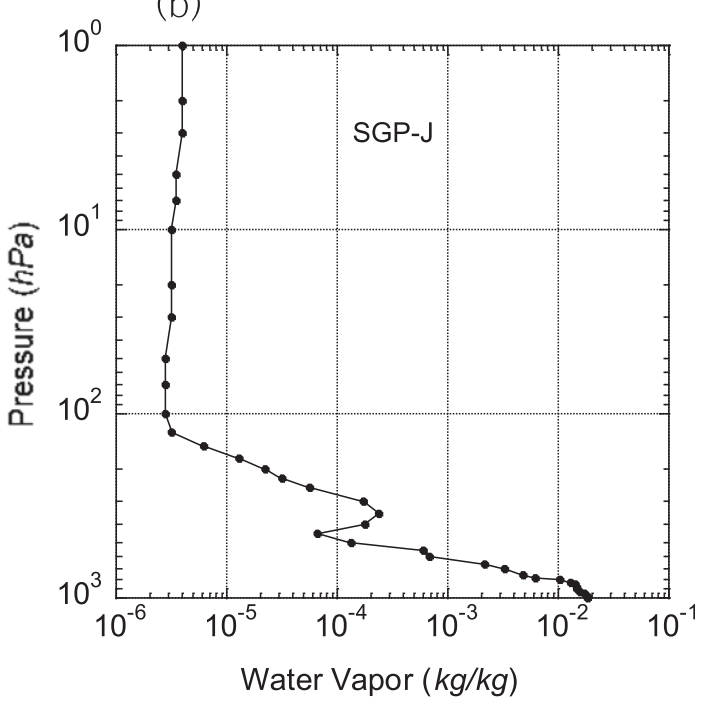

FIG. 8. (a) Temperature profiles of the SGP-J and NSA-J atmospheres. (b) Water vapor profile of the SGP-J atmosphere.

calculated using the LBL method. Therefore, an optimal $\bar{k}_{g}$ cannot be derived separately for individual $g$ groups by minimizing the difference between the correlated- $k$ distribution calculations and the LBL calculations.

There are a number of important issues in the LW radiative transfer parameterization that are not addressed in this study. These issues include the scattering by clouds and aerosols, continuum absorption, the weak absorption lines outside the spectral bands of those gases given in Table 1, and the LBL calculations. Rigorous calculations of scattering by particles are extremely expensive especially for overlapping absorption due to multiple gases, and highly simplified parameterizations are necessary for repeated calculations in an atmospheric model, such as the parameterization

TABLE 9. The mean, root-mean-square (RMS), and maximum ( $\max )$ of the difference between the KD and LBL calculations in the outgoing longwave radiation (OLR; $\mathrm{W} \mathrm{m}^{-2}$ ) and the downward flux at the surface (SFC; $\mathrm{W} \mathrm{m}^{-2}$ ) of the eight sample atmospheres. See the text for the temperature and gas vertical distributions of the eight sample atmospheres. The maximum is the largest deviation of the KD value from the LBL value, so it can be either positive or negative.

\begin{tabular}{lrrrrrrr}
\hline & \multicolumn{3}{c}{ OLR } & & \multicolumn{3}{c}{ SFC } \\
\cline { 2 - 3 } \cline { 6 - 7 } Gas & Mean & RMS & Max & & Mean & RMS & Max \\
\hline $\mathrm{H}_{2} \mathrm{O}$ & -0.20 & 0.31 & 0.64 & & 0.28 & 1.69 & 2.68 \\
$\mathrm{CO}_{2}$ & -0.67 & 0.71 & 0.90 & & 0.66 & 0.67 & 0.78 \\
$\mathrm{O}_{3}$ & -0.35 & 0.36 & 0.53 & & -0.06 & 0.09 & 0.13 \\
$\mathrm{~N}_{2} \mathrm{O}$ & -0.08 & 0.14 & 0.19 & & -0.04 & 0.05 & 0.10 \\
$\mathrm{CH}_{4}$ & 0.01 & 0.02 & 0.04 & & -0.19 & 0.21 & 0.28 \\
\hline
\end{tabular}

developed by Chou et al. (1999) that the effect of the scattering is taken into account by scaling the optical thickness, and the LW radiative transfer is treated as nonscattering. For the continuum absorption, comprehensive schemes are already available, such as the model RRTMG (Mlawer et al. 1997). For the weak absorption line outside the spectral bands of individual gases given Table 1, the absorption is weak and can be treated as gray. Finally, the parameterization is based on data derived from a line-by-line radiative transfer model, which by itself has uncertainties. All of these issues are not directly related to the $k$-distribution approximation and hence beyond the scope of this study.

Acknowledgments. This research was supported by the Ministry of Science and Technology, Taiwan, under the Contracts MOST 108-3116-F-005-001, MOST 1072119-M-001-011 and MOST 108-2111-M-001-016, and the Korean Meteorological Administration Research and Development program under Grant KMI 2018-05910. The HITRAN 2012 molecular spectroscopic database freely available for use in this research is greatly appreciated. Data availability statement: The modern $\mathrm{CO}_{2}, \mathrm{~N}_{2} \mathrm{O}$, and $\mathrm{CH}_{4}$ concentrations in the atmosphere observed at Mauna Loa Observatory were taken from the U.S. Department of Commerce/NOAA website: https://www.esrl.noaa.gov/gmd/ $\mathrm{dv} / \mathrm{iadv} / \mathrm{graph} . \mathrm{php}$ ? code $=\mathrm{MLO} \&$ program $=$ hats $\&$ type $=$ ts. The temperature and humidity data of the eight sample atmospheres were taken from the ECMWF ERA5 reanalysis website: https://www.ecmwf.int/en/forecasts/ datasets/reanalysis-datasets/era5, and the ozone data 
from NASA website: https://doi.org/10.5067/Aura/ OMI/DATA2026.

\section{REFERENCES}

Ambartzumian, V., 1936: The effect of absorption lines on the radiative equilibrium of the outer layers of the stars. Publ. Obs. Astron. Univ. Leningrad, 6, 7-18.

Arking, A., and K. Grossman, 1972: The influence of line shape and band structure on temperatures in planetary atmospheres. J. Atmos. Sci., 29, 937-949, https://doi.org/10.1175/ 1520-0469(1972)029<0937:TIOLSA > 2.0.CO;2.

Chou, M.-D., and A. Arking, 1980: Computation of infrared cooling rates in the water vapor bands. J. Atmos. Sci., 37, 855-867, https://doi.org/10.1175/1520-0469(1980)037<0855: COICRI $>2.0 . \mathrm{CO} ; 2$.

- and L. Kouvaris, 1986: Monochromatic calculations of atmospheric radiative transfer due to molecular line absorption. J. Geophys. Res., 91, 4047-4055, https://doi.org/10.1029/ JD091iD03p04047.

, and K.-T. Lee, 2005: A parameterization of the effective layer emission for infrared radiation calculations. J. Atmos. Sci., 62, 531-541, https://doi.org/10.1175/JAS-3379.1.

— - M. J. Suarez, C. H. Ho, M. M. H. Yan, and K. T. Lee, 1998: Parameterizations for cloud overlapping and shortwave singlescattering properties for use in general circulation and cloud ensemble models. J. Climate, 11, 202-214, https://doi.org/ 10.1175/1520-0442(1998)011<0202:PFCOAS > 2.0.CO;2.

—, K.-T. Lee, S.-C. Tsay, and Q. Fu, 1999: Parameterization for cloud longwave scattering for use in atmospheric models. J. Climate, 12, 159-169, https://doi.org/10.1175/1520-0442-12.1.159.

— M. J. Suarez, X. Z. Liang, and M. M.-H. Yan, 2003: A thermal infrared radiation parameterization for atmospheric studies. NASA Tech. Rep. NASA/TM-2001-104606, Vol. 19, 56 pp., https:// ntrs.nasa.gov/archive/nasa/casi.ntrs.nasa.gov/20010072848.pdf.

Clough, S. A., F. X. Kneizys, and R. W. Davies, 1989: Line shape and the water vapor continuum. Atmos. Res., 23, 229-241, https://doi.org/10.1016/0169-8095(89)90020-3.

— M. J. Iacono, and J.-L. Moncet, 1992: Line-by-line calculations of atmospheric fluxes and cooling rates: Application to water vapor. J. Geophys. Res., 97, 15761-15785, https:// doi.org/10.1029/92JD01419.

Copernicus Climate Change Service, 2017: ERA5: Fifth generation of ECMWF atmospheric reanalyses of the global climate. Copernicus Climate Change Service Climate Data Store, accessed 25 November 2019, https://cds.climate.copernicus.eu/cdsapp\#!/home.

Cusack, S., J. M. Edwards, and J. M. Crowther, 1999: Investigating $k$ distribution methods for parameterizing gaseous absorption in the Hadley Centre climate model. J. Geophys. Res., 104, 2051-2057, https://doi.org/10.1029/1998JD200063.
Fomin, B. A., 2004: A k-distribution technique for radiative transfer simulation in inhomogeneous atmosphere: 1. FKDM, fast k-distribution model for the longwave. J. Geophys. Res., 109, D02110, https://doi.org/10.1029/2003JD003802.

$\mathrm{Fu}$, Q., and K.-N. Liou, 1992: On the correlated $k$-distribution method for radiative transfer in nonhomogeneous atmospheres. J. Atmos. Sci., 49, 2139-2156, https://doi.org/10.1175/ 1520-0469(1992)049<2139:OTCDMF>2.0.CO;2.

Goody, R. M., R. West, L. Chen, and D. Crisp, 1989: The correlated- $k$ method for radiation calculation in nonhomogeneous atmospheres. J. Quant. Spectrosc. Radiat. Transfer, 42, 539550, https://doi.org/10.1016/0022-4073(89)90044-7.

Haan, J. D., and P. Veefkind, 2009: OMI/Aura Ozone (O3) Profile 1-orbit L2 swath $13 \times 48 \mathrm{~km}$ V003. Goddard Earth Sciences Data and Information Services Center, accessed 29 January 2020, https://doi.org/10.5067/Aura/OMI/DATA2026.

Lacis, A., and V. Oinas, 1991: A description of the correlated $k$-distribution method for modeling nongray gaseous absorption, thermal emission, and multiple scattering in vertically inhomogeneous atmospheres. J. Geophys. Res., 96, 9027-9063, https://doi.org/10.1029/90JD01945.

Liou, K.-N., 2002: An Introduction to Atmospheric Radiation. 2nd ed. Academic Press, 583 pp.

McClatchey, R. A., R. W. Fenn, J. E. A. Selby, F. E. Volz, and J. S. Garing, 1972: Optical properties of the atmosphere. Air Force Rep. AFCRL-72-0497, 108 pp.

Mlawer, E. J., S. J. Taubman, P. D. Brown, M. J. Iacono, and S. A. Clough, 1997: Radiative transfer for inhomogeneous atmospheres: RRTM, a validated correlated- $k$ model for the longwave. J. Geophys. Res., 102, 16 663-16 682, https://doi.org/ 10.1029/97JD00237.

Nakajima, T., M. Tsukamoto, Y. Tsushima, A. Numaguti, and T. Kimura, 2000: Modeling of the radiative process in an atmospheric general circulation model. Appl. Opt., 39, 48694878, https://doi.org/10.1364/AO.39.004869.

Rothman, L. S., and Coauthors, 2013: The HITRAN2012 molecular spectroscopic database. J. Quant. Spectrosc. Radiat. Transfer, 130, 4-50, https://doi.org/10.1016/j.jqsrt.2013.07.002.

Seinfeld, J. H., and S. N. Pandis, 2006: Atmospheric Chemistry and Physics: From Air Pollution to Climate Change. 2nd ed. John Wiley and Sons, $1152 \mathrm{pp}$.

Tsai, T. R., R. A. Rose, D. Weidmann, and G. Wysocki, 2012: Atmospheric vertical profiles of $\mathrm{O}_{3}, \mathrm{~N}_{2} \mathrm{O}, \mathrm{CH}_{4}, \mathrm{CCl}_{2} \mathrm{~F}_{2}$, and $\mathrm{H}_{2} \mathrm{O}$ retrieved from external-cavity quantum-cascade laser heterodyne radiometer measurements. Appl. Opt., 51, 87798792, https://doi.org/10.1364/AO.51.008779.

Zhou, M., and Coauthors, 2019: Retrieval of atmospheric $\mathrm{CH}_{4}$ vertical information from ground-based FTS near-infrared spectra. Atmos. Meas. Tech., 12, 6125-6141, https://doi.org/ 10.5194/amt-12-6125-2019. 